\title{
OPEN Lactobacillus reuteri AN417 cell-free culture supernatant as a novel antibacterial agent targeting oral pathogenic bacteria
}

\author{
Kyung MiYang ${ }^{1,3}$, Ji-Sun Kim ${ }^{2,3}$, Hye-Sung Kim ${ }^{1}$, Young-Youn $\mathrm{Kim}^{1}{ }^{1}$, Jeong-Kyu Oh${ }^{1}$, \\ Hye-Won Jung ${ }^{1}$, Doo-Sang Park ${ }^{2 \bowtie}$ \& Kwang-Hak Bae ${ }^{1 \bowtie}$
}

Lactobacillus reuteri AN417 is a newly characterized probiotic strain. The activity of AN417 against oral pathogenic bacteria is unknown. We investigated the antibacterial activity of cell-free $L$. reuteri AN417 culture supernatant (LRS) against three oral pathogens: Porphyromonas gingivalis, Fusobacterium nucleatum, and Streptococcus mutans. $P$. gingivalis and $F$. nucleatum have been implicated in periodontal disease, whereas $S$. mutans causes dental caries. Exposing these oral pathogenic bacteria to LRS significantly reduced their growth rates, intracellular ATP levels, cell viability, and time-to-kill. The minimal inhibitory volume of LRS was $10 \%$ (v/v) against $P$. gingivalis, $20 \%(\mathrm{v} / \mathrm{v})$ for F. nucleatum, and $30 \%(\mathrm{v} / \mathrm{v})$ for $S$. mutans. LRS significantly reduced the integrity of biofilms and significantly suppressed the expression of various genes involved in $P$. gingivalis biofilm formation. The $L$. reuteri AN417 genome lacked genes encoding reuterin, reuteran, and reutericyclin, which are major antibacterial compounds produced in L. reuteri strains. LRS treated with lipase and $\alpha$-amylase displayed decreased antibacterial activity against oral pathogens. These data suggest that the antibacterial substances in LRS are carbohydrates and/or fatty acid metabolites. Our results demonstrate that LRS has antimicrobial activity against dental pathogenic bacteria, highlighting its potential utility for the prevention and treatment of $P$. gingivalis periodontal disease.

Periodontal disease is a chronic inflammatory disease caused by the accumulation of different pathogenic biofilmforming bacteria in dental pockets ${ }^{1}$. Biofilms that develop on tooth surfaces contain oral microbes, and the formation of bacterial plaque causes gingivitis with redness and swelling of the gingiva ${ }^{2}$. Periodontitis refers to an irreversible loss of adhesion, in which tissues such as periodontal ligaments, alveolar bone, and chalk that support teeth are absorbed as the inflammatory response of the tissues to pathogenic bacterial stimulation intensifies ${ }^{3}$. Collagen fibers in the periodontal ligament are destroyed, forming a periodontal sac between the gingiva and the teeth, deepening through the absorption of the alveolar bone $\mathrm{s}^{3,4}$. This creates an ecological environment that favors the propagation of various anaerobic bacteria, causing periodontal disease ${ }^{5}$. Endotoxins or metabolites formed by microorganisms participate in tissue breakthrough processes by increasing the secretion of pro-inflammatory cytokines from tissues and immune cells ${ }^{6}$.

The pathogens most known to be associated with the development of oral diseases are Porphyromonas gingivalis, Fusobacterium nucleatum, and Streptococcus mutans. Among oral pathogens, P. gingivalis and F. nucleatum are Gram-negative and obligate anaerobes that cause periodontal diseases ${ }^{7,8}$. These two oral pathogens degrade collagen, induce halitosis, and produce endotoxins, such as lipopolysaccharides (LPS), which destroy alveolar bone and cause tooth loss ${ }^{9}$. S. mutans produces acidic compounds and plays an important role in the formation of biofilms on teeth, which cause dental caries ${ }^{10}$. These oral pathogens can penetrate directly into the vascular endothelial cells or enter damaged blood vessels and adhere to specific organs, ultimately leading to systemic disease $\mathrm{e}^{11}$.

Substances produced by lactic acid bacteria during the metabolism of prebiotics (dietary fiber) can be beneficial to human health ${ }^{12}$. In a rat model, the administration of live probiotic Bifidobacteria was reported to protect against periodontal destruction and to decrease inflammatory intermediates in rats with ligature-induced

${ }^{1}$ Institute of Biomedical Science, Apple Tree Dental Hospital, 1450, Jungang-ro, Ilsanseo-gu, Goyang-si, Gyeonggi-do 10387, Republic of Korea. ${ }^{2}$ Biological Resources Center, Korea Research Institute of Bioscience \& Biotechnology (KRIBB), Jeong-up 56212, Republic of Korea. ${ }^{3}$ These authors contributed equally: Kyung Mi Yang and Ji-Sun Kim. ${ }^{\boxplus}$ email: dspark@kribb.re.kr; sadent@naver.com 
periodontitis ${ }^{13}$. A recent review highlighted potential antimicrobial agents, including those produced by lactic acid bacteria, for the treatment of dental diseases ${ }^{4}$.

Although antibiotics are still widely used to treat oral diseases, they cause side effects, such as the generation of resistant strains and microbial substitution ${ }^{14}$. Thus, a variety of natural substances that have few side effects are being used proactively. Numerous ongoing studies are examining the antimicrobial activities of plant extracts that inhibit the growth of oral pathogenic bacteria ${ }^{15-17}$. Adjuvant treatments using ozone are also being explored as a new approach for managing chronic periodontitis ${ }^{18}$.

Recent data have indicated the potential value of probiotic microorganisms in oral health ${ }^{19}$. For example, cellfree culture supernatants of a Weissella cibaria strain showed antibacterial activity against periodontal pathogens. The effects were dependent on either the acidity of the supernatant or the level of hydrogen peroxide produced by $W$. cibaria ${ }^{20}$. Another study reported antimicrobial and antibiofilm activities of Lactobacillus kefiranofaciens culture supernatants against oral pathogens. Heat-killed $L$. reuteri and supernatants from cell-free $L$. reuteri cultures had antibacterial effects on P. gingivalis that were similar to those of live L. reuteri cells, although in an invertebrate model, hemocyte density was significantly increased only in the presence of live L. reuteri and not with the culture supernatant or heat-killed cells ${ }^{21}$. During fermentation, lactic acid bacteria produce metabolites, such as extracellular polymeric substances, functional proteins, and peptides, which are bioactive compounds with known beneficial effects on human health ${ }^{22}$. The antimicrobial effect of the supernatant of lactic acid bacteria (LAB) was not after treatment with proteinase $\mathrm{K}$, pepsin, and papain ${ }^{23}$.

This study evaluated the antibacterial potential of a new probiotic strain, L. reuteri AN417, to improve oral health. Our findings indicate that this strain has potential as a therapeutic agent for the treatment of chronic periodontitis and the prevention of dental caries.

\section{Results}

Isolation and characterization of $L$. reuteri strains. We isolated 135 L. reuteri strains from human infants and 6-month-old female swine under anaerobic conditions. Bacterial isolates were identified by $16 \mathrm{~S}$ rRNA gene sequencing and a matrix-assisted laser desorption/ionization time of flight (MALDI-TOF) biotyper (Bruker, Table 1). Primary screening for antimicrobial activity against pathogens, including Escherichia coli (KCTC 2571), Pseudomonas aeruginosa (DSM 50071), and S. mutans (KCTC 3065), was performed to select L. reuteri strains that had exhibited antimicrobial activity against periodontopathic bacteria in a disk diffusion assay (Fig. 1A). The results showed that L. reuteri AN417 displayed the strongest antibacterial activity against pathogens.

In addition, the production of 1, 3-propanediol (1,3-PDO) by the isolated $L$. reuteri strains was determined using a high-performance liquid chromatography (HPLC) system (Table 1 and Supplementary Fig. S1A). The 1,3-PDO is valuable compound used as a replacement for petroleum-based glycols, including propylene glycol, butylene glycol, and glycerin. It has been reported that L. reuteri metabolizes glycerol to reuterin (3-hydroxypropionaldehyde, 3-HPA) and then converts reuterin to 1,3-PDO. L. reuteri cannot grow on glycerol as the sole carbon source and the conversion to 1, 3-PDO from glycerol requires NADH produced by glucose metabolism. Therefore, we tested the production level of 1, 3-PDO under co-fermentation in the presence of both glycerol and glucose in the culture medium.

Interestingly, most (but not all) L. reuteri isolates originating from swine did not produce 1, 3-PDO, whereas every strain of human origin did (Table 1). Genomic analysis showed that L. reuteri AN417 isolated from swine did not encode glycerol dehydratase (dhaB), which catalyzes 3-HPA production, and 1,3-propanediol dehydrogenases $(d h a T)$, which catalyze 1,3-PDO production. The data implied that the L reuteri strains exhibit hostspecific characteristics in metabolite production.

Potentially important inhibitory activity of L. reuteri AN417 supernatant (LRS) against oral bacterial pathogens. When the antimicrobial activity of the newly identified L. reuteri strains was assessed, we observed that L. reuteri AN417 culture supernatant (LRS) outperformed those of our other tested strains. We first observed whether isolated L. reuteri strains affected the growth of the oral pathogens $S$. mutans KCTC 3065 and P. gingivalis BAA-308 (Fig. 1A, B). The clear zone was the largest when S. mutans was treated with LRS compared to the supernatants from other strains. Moreover, the growth of $P$. gingivalis was more highly inhibited when $10 \%(\mathrm{v} / \mathrm{v})$ LRS was added to the medium than when supernatants derived from other $L$. reuteri strains were treated. Furthermore, growth of $P$. gingivalis was most inhibited when $10 \%(\mathrm{v} / \mathrm{v})$ LRS was added to the medium (Fig. 1B). The findings indicated that cell-free culture supernatant derived from L. reuteri AN417 exhibited the highest activity against $P$. gingivalis and potentially against other oral pathogenic bacteria.

Next, we determined that the antimicrobial bioactive substances are present in the culture supernatant, not inside the bacterial cells themselves. To establish whether the active substances were present in the culture supernatant or in the bacterial cells, bacterial cell extracts (BE) were prepared using ethyl acetate. BE and LRS, which were concentrated and diluted to the desired values, were treated with $P$. gingivalis for $24 \mathrm{~h}$. Compared with the control treated only with HEPES or de Man Rogosa and Sharpe (MRS), LRS substantially inhibited the growth of $P$. gingivalis. However, no effect was observed with the BE treatment. Furthermore, compared with control treatments, LRS significantly reduced $P$. gingivalis intracellular ATP levels, whereas the BE treatment did not (Fig. 1C). Administration of $20 \%$ or $40 \%$ (v/v) LRS for $96 \mathrm{~h}$ significantly reduced ATP levels and growth of the pathogenic bacteria (Fig. 1D).

LRS inhibits the growth of oral pathogenic bacteria. The inhibitory effects of $10 \%, 20 \%, 30 \%$, and $40 \%(\mathrm{v} / \mathrm{v})$ LRS on the growth of selected oral pathogenic bacteria (P. gingivalis, F. nucleatum, and S. mutans) were assessed. As the results, growth inhibition against tested pathogens was dependent on LRS concentrations. Treat- 


\begin{tabular}{|c|c|c|c|c|c|c|c|}
\hline No & ID & Strain & Origin & $\begin{array}{l}\text { Residual } \\
\text { glucose }(\mathrm{g} / \mathrm{L})\end{array}$ & \begin{tabular}{|l} 
Residual \\
glycerol $(\mathrm{g} / \mathrm{L})$
\end{tabular} & $\begin{array}{l}\text { 1,3-PDO } \\
\text { production }(\mathrm{g} / \mathrm{L})^{\mathrm{a}}\end{array}$ & \begin{tabular}{|l|}
$\begin{array}{l}\text { Reuterin } \\
\text { production }\end{array}$ \\
\end{tabular} \\
\hline 1 & PB3 & Lactobacillus reuteri & Swine feces & 1.96 & 6.93 & ++ & + \\
\hline 2 & PB6 & Lactobacillus reuteri & Swine feces & 0.16 & 5.51 & +++ & + \\
\hline 3 & PF4 & Lactobacillus reuteri & Swine feces & 1.18 & 4.96 & +++ & - \\
\hline 4 & PPF3 & Lactobacillus reuteri & Swine feces & 2.76 & 8.10 & ++ & - \\
\hline 5 & PMA2 & Lactobacillus reuteri & Swine feces & 0.05 & 21.49 & - & - \\
\hline 6 & PMF1 & Lactobacillus reuteri & Swine feces & 0.64 & 20.99 & - & - \\
\hline 7 & PMF2 & Lactobacillus reuteri & Swine feces & 0.57 & 21.45 & - & - \\
\hline 8 & AN306 & Lactobacillus reuteri & Small intestine of swine & 0.35 & 21.47 & - & - \\
\hline 9 & AN313 & Lactobacillus reuteri & Small intestine of swine & 0.56 & 20.84 & - & - \\
\hline 10 & AN403 & Lactobacillus reuteri & Small intestine of swine & 4.51 & 21.47 & - & - \\
\hline 11 & AN413 & Lactobacillus reuteri & Small intestine of swine & 0.05 & 6.05 & - & - \\
\hline 12 & AN417 & Lactobacillus reuteri & Small intestine of swine & 1.08 & 20.40 & - & - \\
\hline 13 & AN507 & Lactobacillus reuteri & Small intestine of swine & 0.45 & 20.09 & - & - \\
\hline 14 & AN509 & Lactobacillus reuteri & Small intestine of swine & 0.08 & 21.43 & - & - \\
\hline 15 & AN510 & Lactobacillus reuteri & Small intestine of swine & 2.21 & 21.50 & - & - \\
\hline 16 & AN511 & Lactobacillus reuteri & Small intestine of swine & 0.28 & 21.42 & - & - \\
\hline 17 & AN513 & Lactobacillus reuteri & Small intestine of swine & 5.19 & 21.26 & - & - \\
\hline 18 & AN516 & Lactobacillus reuteri & Small intestine of swine & 1.39 & 21.48 & - & - \\
\hline 19 & AN519 & Lactobacillus reuteri & Small intestine of swine & 0.20 & 21.30 & - & - \\
\hline 20 & AN521 & Lactobacillus reuteri & Small intestine of swine & 0.18 & 21.17 & - & - \\
\hline 21 & AN523 & Lactobacillus reuteri & Small intestine of swine & 1.23 & 21.30 & - & - \\
\hline 22 & AN525 & Lactobacillus reuteri & Small intestine of swine & 0.13 & 21.28 & - & - \\
\hline 23 & AN527 & Lactobacillus reuteri & Small intestine of swine & 0.12 & 20.95 & - & - \\
\hline 24 & AN530 & Lactobacillus reuteri & Small intestine of swine & 0.46 & 21.21 & - & - \\
\hline 25 & AN540 & Lactobacillus reuteri & Small intestine of swine & 1.00 & 21.36 & - & - \\
\hline 26 & AN543 & Lactobacillus reuteri & Small intestine of swine & 0.10 & 21.42 & - & - \\
\hline 27 & AN546 & Lactobacillus reuteri & Small intestine of swine & 3.58 & 21.42 & - & - \\
\hline 28 & AN548 & Lactobacillus reuteri & Small intestine of swine & 0.24 & 21.32 & - & - \\
\hline 29 & AN703 & Lactobacillus reuteri & Small intestine of swine & 8.13 & 21.46 & - & - \\
\hline 30 & AN704 & Lactobacillus reuteri & Small intestine of swine & 0.42 & 21.37 & - & - \\
\hline 31 & AN705 & Lactobacillus reuteri & Small intestine of swine & 1.38 & 20.89 & - & - \\
\hline 32 & AN708 & Lactobacillus reuteri & Small intestine of swine & 5.99 & 21.38 & - & - \\
\hline 33 & AN709 & Lactobacillus reuteri & Small intestine of swine & 3.36 & 21.42 & - & - \\
\hline 34 & AN711 & Lactobacillus reuteri & Small intestine of swine & 2.64 & 21.41 & - & - \\
\hline 35 & AN722 & Lactobacillus reuteri & Small intestine of swine & 4.81 & 21.24 & - & - \\
\hline 36 & AN724 & Lactobacillus reuteri & Small intestine of swine & 0.12 & 21.34 & - & - \\
\hline 37 & AN727 & Lactobacillus reuteri & Small intestine of swine & 2.48 & 21.22 & - & - \\
\hline 38 & AN832 & Lactobacillus reuteri & Small intestine of swine & 7.69 & 21.39 & - & - \\
\hline 39 & AN903 & Lactobacillus reuteri & Small intestine of swine & 1.03 & 21.47 & - & - \\
\hline 40 & AN904 & Lactobacillus reuteri & Small intestine of swine & 3.63 & 21.49 & - & - \\
\hline 41 & AN906 & Lactobacillus reuteri & Small intestine of swine & 9.73 & 18.93 & - & - \\
\hline 42 & AN919 & Lactobacillus reuteri & Small intestine of swine & 12.51 & 18.90 & - & - \\
\hline 43 & AN924 & Lactobacillus reuteri & Small intestine of swine & 7.59 & 18.78 & - & - \\
\hline 44 & AN925 & Lactobacillus reuteri & Small intestine of swine & 9.31 & 19.01 & - & - \\
\hline 45 & AN926 & Lactobacillus reuteri & Small intestine of swine & 9.90 & 21.05 & - & - \\
\hline 46 & AN933 & Lactobacillus reuteri & Small intestine of swine & 4.88 & 19.54 & - & - \\
\hline 47 & AN941 & Lactobacillus reuteri & Small intestine of swine & 10.13 & 19.27 & - & - \\
\hline 48 & AN943 & Lactobacillus reuteri & Small intestine of swine & 0.75 & 20.79 & - & - \\
\hline 49 & AN947 & Lactobacillus reuteri & Small intestine of swine & 9.92 & 18.72 & - & + \\
\hline 50 & AN1002 & Lactobacillus reuteri & Small intestine of swine & 12.09 & 19.00 & - & - \\
\hline 51 & AN1009 & Lactobacillus reuteri & Small intestine of swine & 9.69 & 18.99 & - & - \\
\hline 52 & AN1016 & Lactobacillus reuteri & Small intestine of swine & 4.47 & 20.87 & - & - \\
\hline 53 & AN1019 & Lactobacillus reuteri & Small intestine of swine & 8.83 & 20.52 & - & - \\
\hline 54 & AN1026 & Lactobacillus reuteri & Small intestine of swine & 7.55 & 19.04 & - & - \\
\hline 55 & AN1033 & Lactobacillus reuteri & Small intestine of swine & 2.38 & 20.96 & - & - \\
\hline 56 & AN1034 & Lactobacillus reuteri & Small intestine of swine & 5.58 & 19.42 & - & - \\
\hline \multicolumn{8}{|c|}{ Continued } \\
\hline
\end{tabular}




\begin{tabular}{|c|c|c|c|c|c|c|c|}
\hline No & ID & Strain & Origin & $\begin{array}{l}\text { Residual } \\
\text { glucose }(\mathrm{g} / \mathrm{L})\end{array}$ & \begin{tabular}{|l} 
Residual \\
glycerol (g/L)
\end{tabular} & $\begin{array}{l}\text { 1,3-PDO } \\
\text { production }(\mathrm{g} / \mathrm{L})^{\mathrm{a}}\end{array}$ & $\begin{array}{l}\text { Reuterin } \\
\text { production }^{\mathrm{b}}\end{array}$ \\
\hline 57 & AN1109 & Lactobacillus reuteri & Small intestine of swine & 8.60 & 21.03 & - & - \\
\hline 58 & SBF301 & Lactobacillus reuteri & Infant feces & 0.51 & 5.70 & ++ & + \\
\hline 59 & SBF302 & Lactobacillus reuteri & Infant feces & 1.30 & 7.37 & ++ & + \\
\hline 60 & SBF303 & Lactobacillus reuteri & Infant feces & 1.04 & 7.09 & ++ & ++ \\
\hline 61 & SBF304 & Lactobacillus reuteri & Infant feces & 2.31 & 8.33 & ++ & + \\
\hline 62 & SBF305 & Lactobacillus reuteri & Infant feces & 10.85 & 15.80 & + & + \\
\hline 63 & SBF306 & Lactobacillus reuteri & Infant feces & 0.32 & 5.98 & +++ & + \\
\hline 64 & SBF307 & Lactobacillus reuteri & Infant feces & 0.59 & 6.27 & +++ & ++ \\
\hline 65 & SBF309 & Lactobacillus reuteri & Infant feces & 3.99 & 9.26 & ++ & - \\
\hline 66 & SBF310 & Lactobacillus reuteri & Infant feces & 3.57 & 9.09 & ++ & + \\
\hline 67 & SBF311 & Lactobacillus reuteri & Infant feces & 0.08 & 5.20 & ++ & ++ \\
\hline 68 & SBF312 & Lactobacillus reuteri & Infant feces & 4.47 & 10.15 & ++ & + \\
\hline 69 & SBF313 & Lactobacillus reuteri & Infant feces & 7.43 & 12.87 & ++ & + \\
\hline 70 & SBF314 & Lactobacillus reuteri & Infant feces & 5.78 & 11.30 & ++ & + \\
\hline 71 & SBF315 & Lactobacillus reuteri & Infant feces & 4.40 & 9.93 & ++ & + \\
\hline 72 & SBF316 & Lactobacillus reuteri & Infant feces & 1.33 & 8.92 & ++ & - \\
\hline 73 & SBF317 & Lactobacillus reuteri & Infant feces & 12.47 & 16.74 & + & - \\
\hline 74 & SBF318 & Lactobacillus reuteri & Infant feces & 5.78 & 11.44 & ++ & + \\
\hline 75 & SBF320 & Lactobacillus reuteri & Infant feces & 11.91 & 16.46 & + & + \\
\hline 76 & SBF321 & Lactobacillus reuteri & Infant feces & 9.37 & 14.92 & + & + \\
\hline 77 & SBF325 & Lactobacillus reuteri & Infant feces & 0.32 & 5.27 & +++ & + \\
\hline 78 & SBF326 & Lactobacillus reuteri & Infant feces & 6.18 & 11.47 & ++ & + \\
\hline 79 & SBF327 & Lactobacillus reuteri & Infant feces & 5.54 & 10.86 & ++ & +++ \\
\hline 80 & SBF328 & Lactobacillus reuteri & Infant feces & 0.95 & 14.94 & + & +++ \\
\hline 81 & SBF329 & Lactobacillus reuteri & Infant feces & 1.13 & 7.73 & +++ & - \\
\hline 82 & SBF330 & Lactobacillus reuteri & Infant feces & 8.63 & 14.70 & + & + \\
\hline 83 & SBF331 & Lactobacillus reuteri & Infant feces & 0.39 & 6.30 & +++ & ++ \\
\hline 84 & SBF332 & Lactobacillus reuteri & Infant feces & 0.77 & 7.17 & +++ & ++ \\
\hline 85 & SBF333 & Lactobacillus reuteri & Infant feces & 1.59 & 8.07 & +++ & +++ \\
\hline 86 & SBF334 & Lactobacillus reuteri & Infant feces & 1.65 & 8.12 & ++ & ++ \\
\hline 87 & SBF335 & Lactobacillus reuteri & Infant feces & 0.32 & 6.50 & +++ & +++ \\
\hline 88 & SBF336 & Lactobacillus reuteri & Infant feces & 4.58 & 10.57 & ++ & ++ \\
\hline 89 & SBF401 & Lactobacillus reuteri & Infant feces & 0.29 & 6.41 & +++ & + \\
\hline 90 & SBF402 & Lactobacillus reuteri & Infant feces & 1.27 & 9.55 & ++ & ++ \\
\hline 91 & SBF417 & Lactobacillus reuteri & Infant feces & 0.53 & 7.07 & +++ & + \\
\hline 92 & SBF418 & Lactobacillus reuteri & Infant feces & 5.69 & 11.51 & ++ & + \\
\hline 93 & SBF419 & Lactobacillus reuteri & Infant feces & 0.32 & 5.36 & +++ & + \\
\hline 94 & SBF420 & Lactobacillus reuteri & Infant feces & 0.29 & 6.19 & +++ & + \\
\hline 95 & SBF447 & Lactobacillus reuteri & Infant feces & 0.66 & 6.86 & +++ & + \\
\hline 96 & MBF301 & Lactobacillus reuteri & Infant feces & 0.99 & 7.02 & +++ & + \\
\hline 97 & MBF302 & Lactobacillus reuteri & Infant feces & 0.25 & 5.39 & +++ & + \\
\hline 98 & MBF303 & Lactobacillus reuteri & Infant feces & 0.47 & 5.20 & +++ & + \\
\hline 99 & MBF304 & Lactobacillus reuteri & Infant feces & 0.41 & 4.18 & +++ & + \\
\hline 100 & MBF307 & Lactobacillus reuteri & Infant feces & 12.83 & 17.83 & + & + \\
\hline 101 & MBF312 & Lactobacillus reuteri & Infant feces & 1.38 & 7.84 & +++ & + \\
\hline 102 & MBF326 & Lactobacillus reuteri & Infant feces & 0.29 & 5.54 & +++ & + \\
\hline 103 & MBF327 & Lactobacillus reuteri & Infant feces & 7.63 & 13.25 & ++ & + \\
\hline 104 & MBF329 & Lactobacillus reuteri & Infant feces & 12.96 & 17.58 & + & + \\
\hline 105 & MBF335 & Lactobacillus reuteri & Infant feces & 0.26 & 4.86 & +++ & + \\
\hline 106 & MBF342 & Lactobacillus reuteri & Infant feces & 0.25 & 4.87 & +++ & + \\
\hline 107 & MBF344 & Lactobacillus reuteri & Infant feces & 0.27 & 5.55 & +++ & + \\
\hline 108 & MBF351 & Lactobacillus reuteri & Infant feces & 0.27 & 5.49 & +++ & + \\
\hline 109 & MBF355 & Lactobacillus reuteri & Infant feces & 0.25 & 4.77 & +++ & + \\
\hline 110 & MBF356 & Lactobacillus reuteri & Infant feces & 0.28 & 4.76 & +++ & + \\
\hline 111 & MBF358 & Lactobacillus reuteri & Infant feces & 0.49 & 5.88 & +++ & + \\
\hline 112 & MBF359 & Lactobacillus reuteri & Infant feces & 0.62 & 6.25 & +++ & + \\
\hline \multicolumn{8}{|c|}{ Continued } \\
\hline
\end{tabular}




\begin{tabular}{|l|l|l|l|l|l|l|l|}
\hline No & ID & Strain & Origin & $\begin{array}{l}\text { Residual } \\
\text { glucose }(\mathbf{g} / \mathbf{L})\end{array}$ & $\begin{array}{l}\text { Residual } \\
\text { glycerol }(\mathbf{g} / \mathbf{L})\end{array}$ & $\begin{array}{l}\mathbf{1 , 3} \text {-PDO } \\
\text { production }(\mathbf{g} / \mathbf{L})^{\mathbf{a}}\end{array}$ & $\begin{array}{l}\text { Reuterin } \\
\text { production }^{\mathbf{b}}\end{array}$ \\
\hline 113 & MBF483 & Lactobacillus reuteri & Infant feces & 2.82 & 9.16 & ++ & + \\
\hline 114 & MBF484 & Lactobacillus reuteri & Infant feces & 0.26 & 4.76 & +++ & + \\
\hline 115 & MBF496 & Lactobacillus reuteri & Infant feces & 0.24 & 5.71 & +++ & ++ \\
\hline 116 & MBF497 & Lactobacillus reuteri & Infant feces & 0.27 & 4.88 & +++ & + \\
\hline 117 & MBF498 & Lactobacillus reuteri & Infant feces & 0.23 & 4.64 & +++ & + \\
\hline 118 & MBF4112 & Lactobacillus reuteri & Infant feces & 0.67 & 7.28 & +++ & + \\
\hline 119 & MBF4116 & Lactobacillus reuteri & Infant feces & 0.24 & 4.23 & +++ & + \\
\hline 120 & NBF306 & Lactobacillus reuteri & Infant feces & 0.27 & 4.40 & +++ & + \\
\hline 121 & NBF307 & Lactobacillus reuteri & Infant feces & 0.66 & 7.74 & ++ & + \\
\hline 122 & NBF308 & Lactobacillus reuteri & Infant feces & 0.76 & 8.32 & ++ & + \\
\hline 123 & NBF309 & Lactobacillus reuteri & Infant feces & 0.90 & 7.88 & ++ & + \\
\hline 124 & NBF310 & Lactobacillus reuteri & Infant feces & 0.57 & 7.17 & ++ & + \\
\hline 125 & NBF317 & Lactobacillus reuteri & Infant feces & 12.66 & 18.11 & + & + \\
\hline 126 & NBF319 & Lactobacillus reuteri & Infant feces & 5.73 & 12.01 & + & + \\
\hline 127 & NBF320 & Lactobacillus reuteri & Infant feces & 8.06 & 13.97 & + & + \\
\hline 128 & NBF331 & Lactobacillus reuteri & Infant feces & 0.66 & 7.71 & +++ & + \\
\hline 129 & NBF342 & Lactobacillus reuteri & Infant feces & 1.80 & 8.32 & ++ & + \\
\hline 130 & NBF344 & Lactobacillus reuteri & Infant feces & 2.10 & 8.96 & ++ & + \\
\hline 131 & NBF347 & Lactobacillus reuteri & Infant feces & 0.27 & 7.13 & ++ & + \\
\hline 132 & NBF409 & Lactobacillus reuteri & Infant feces & 0.09 & 5.73 & +++ & + \\
\hline 133 & NBF430 & Lactobacillus reuteri & Infant feces & 0.10 & 5.80 & +++ & + \\
\hline 134 & NBF456 & Lactobacillus reuteri & Infant feces & 0.10 & 5.50 & +++ & + \\
\hline 135 & NBF474 & Lactobacillus reuteri & Infant feces & 0.66 & 9.50 & ++ & + \\
\hline
\end{tabular}

Table 1. Isolation of Lactobacillus reuteri strains, and determination of 1,3-PDO and reuterin production. 1,3PDO and reuterin production were determined in Lactobacillus reuteri isolates using HPLC and Colorimetric method, respectively. ${ }^{\mathrm{a}} 1,3-\mathrm{PDO}$ production:+++; $>12 \mathrm{~g} / \mathrm{L},++;>7 \mathrm{~g} / \mathrm{L},+;>0 \mathrm{~g} / \mathrm{L},-;$ not detected. ${ }^{\mathrm{b}}$ Reuterin production:+++;>0.5 at $560 \mathrm{~nm},++;>0.3$ at $560 \mathrm{~nm},+;>0.1$ at $560 \mathrm{~nm},-;$ not detected.

ment with $40 \%(\mathrm{v} / \mathrm{v})$ LRS significantly inhibited the growth of the three pathogenic bacterial strains. In contrast, $10 \%(\mathrm{v} / \mathrm{v})$ LRS did not inhibit the growth of F. nucleatum and S. mutans. However, the growth of P. gingivalis was significantly inhibited in 10, 20 and $40 \%$ of LRS treatment ( $80 \%$ reduction in growth rate) (Fig. 2 A). Prolonged treatment for up to $48 \mathrm{~h}$ with $40 \%(\mathrm{v} / \mathrm{v})$ LRS resulted in a stable continuation of the inhibitory effect in all three strains (Fig. 2B).

LRS effectively reduces the viability of oral pathogenic bacteria. In fluorescent cell-staining assays, LRS increased the number of dead pathogenic bacteria and decreased the number of live pathogenic bacteria. To evaluate the effect of LRS on the viability of the three pathogenic bacterial strains, a mixture of SYTO9 green fluorescence nucleic acid stain and propidium iodide was used for cell staining. The cells were observed by fluorescence microscopy. Compared with the negative control (MRS or Brain Heart Infusion [BHI] medium), the intensity of green fluorescence emitted by live bacteria decreased in P. gingivalis, F. nucleatum, and S. mutans cultures treated with LRS. In S. mutans, the intensity of red fluorescence, indicating dead bacteria, increased with LRS treatment (Fig. 3A). After treatment with LRS, bacterial death was observed over time. As a result, it was observed that $P$. gingivalis died rapidly starting $8 \mathrm{~h}$ after LRS treatment (Fig. 3B). These results were consistent with those of LIVE/DEAD BacLight analysis, with LRS treatment substantially reducing colony forming units compared with the MRS treatment.

Antimicrobial activity of LRS against oral pathogenic bacteria, especially $\boldsymbol{P}$. gingivalis. Treatment of the three oral pathogenic bacteria with LRS revealed a minimum inhibitory volume (MIV) of approximately $10 \%(\mathrm{v} / \mathrm{v})$ for P. gingivalis. The MIV was $20 \%$ and $40 \%(\mathrm{v} / \mathrm{v})$ for F. nucleatum and S. mutans, respectively (Fig. 3C).

LRS impedes biofilm formation by the oral pathogenic bacteria. To confirm the antibiofilm activity of LRS against the biofilm formation during the early stage of bacterial colonization, LRS was added to $P$. gingivalis and $S$. mutans cultures immediately after bacterial inoculations, so that the effects of LRS on biofilm formation during the initial attachment phase could be examined. LRS treatment substantially reduced the fluorescence intensity compared with the control treatment (Fig. 4A), which was consistent with the quantitative results. To determine the concentration of LRS that eradicates established $P$. gingivalis biofilms, biofilms developed for 5 days were treated with LRS and stained with crystal violet. Compared with the control treatment, 

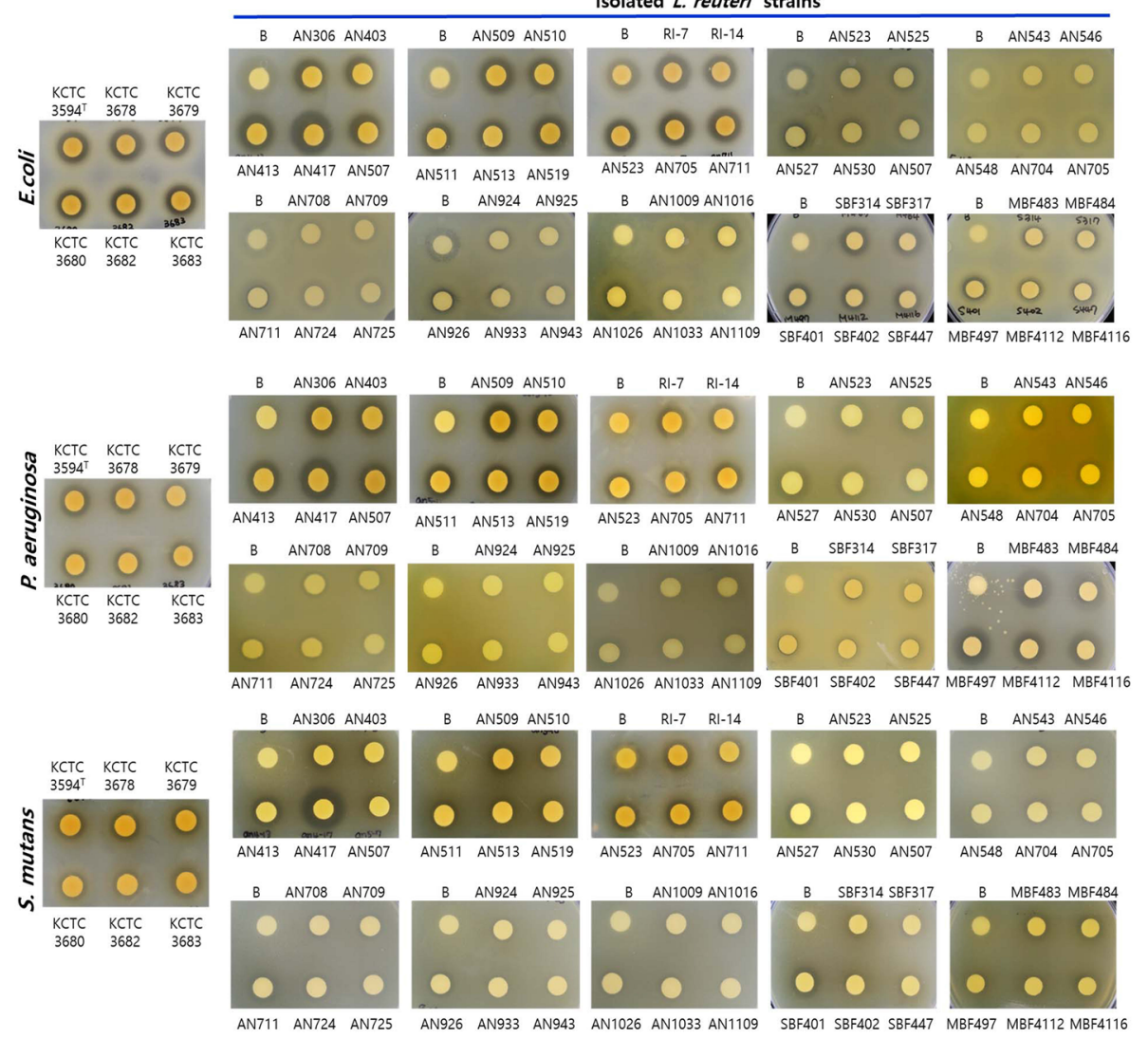

B

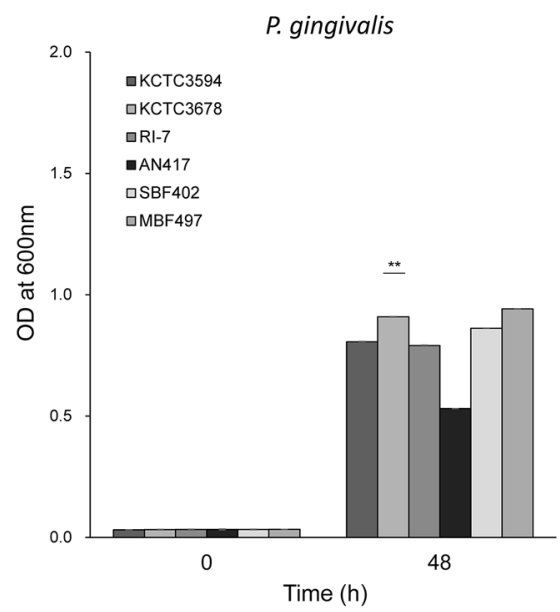

C

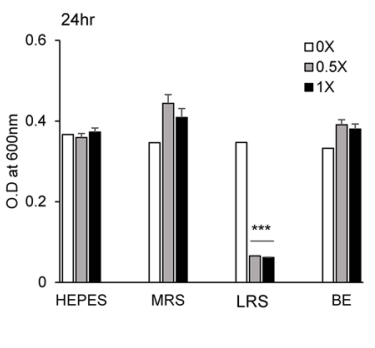

P. gingivalis

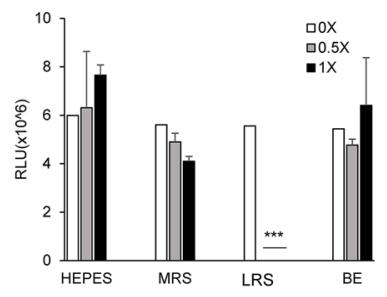

P. gingivalis

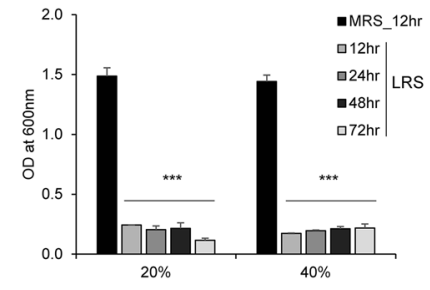

Figure 1. Evaluation of the antimicrobial activity of culture supernatants of L. reuteri strains against E. coli, $P$. aeruginosa, S. mutans, and P. gingivalis. (A) The antimicrobial activity of culture supernatants from newly isolated and reference $L$. reuteri strains against E. coli, P. aeruginosa and S. mutans was analyzed through disk diffusion assays following $24 \mathrm{~h}$ incubation at $37^{\circ} \mathrm{C}$. (B) Growth curve of $P$. gingivalis at $\mathrm{OD}_{600}$ measured using a disposable curvet $48 \mathrm{~h}$ after treatment with the supernatants of each strains. Significant differences from the control $(p<0.01)$ are indicated by ${ }^{* *}$. (C) The effect of various concentrations of LRS and BE on P. gingivalis growth, measured by $\mathrm{OD}_{600}$ (left panel) and intracellular ATP levels (right panel) following a $24 \mathrm{~h}$ incubation at $37^{\circ} \mathrm{C}$. MRS medium was used as the negative control. Significant differences from the control $(p<0.001)$ are indicated by ${ }^{* * *}$. (D) The effect of $20 \%(\mathrm{v} / \mathrm{v})$ and $40 \%(\mathrm{v} / \mathrm{v})$ LRS on $P$. gingivalis growth (left panel) and intracellular ATP levels (right panel) over time. MRS medium was used as the negative control. Significant differences from the control $(p<0.001)$ are indicated by ${ }^{* * *}$. 

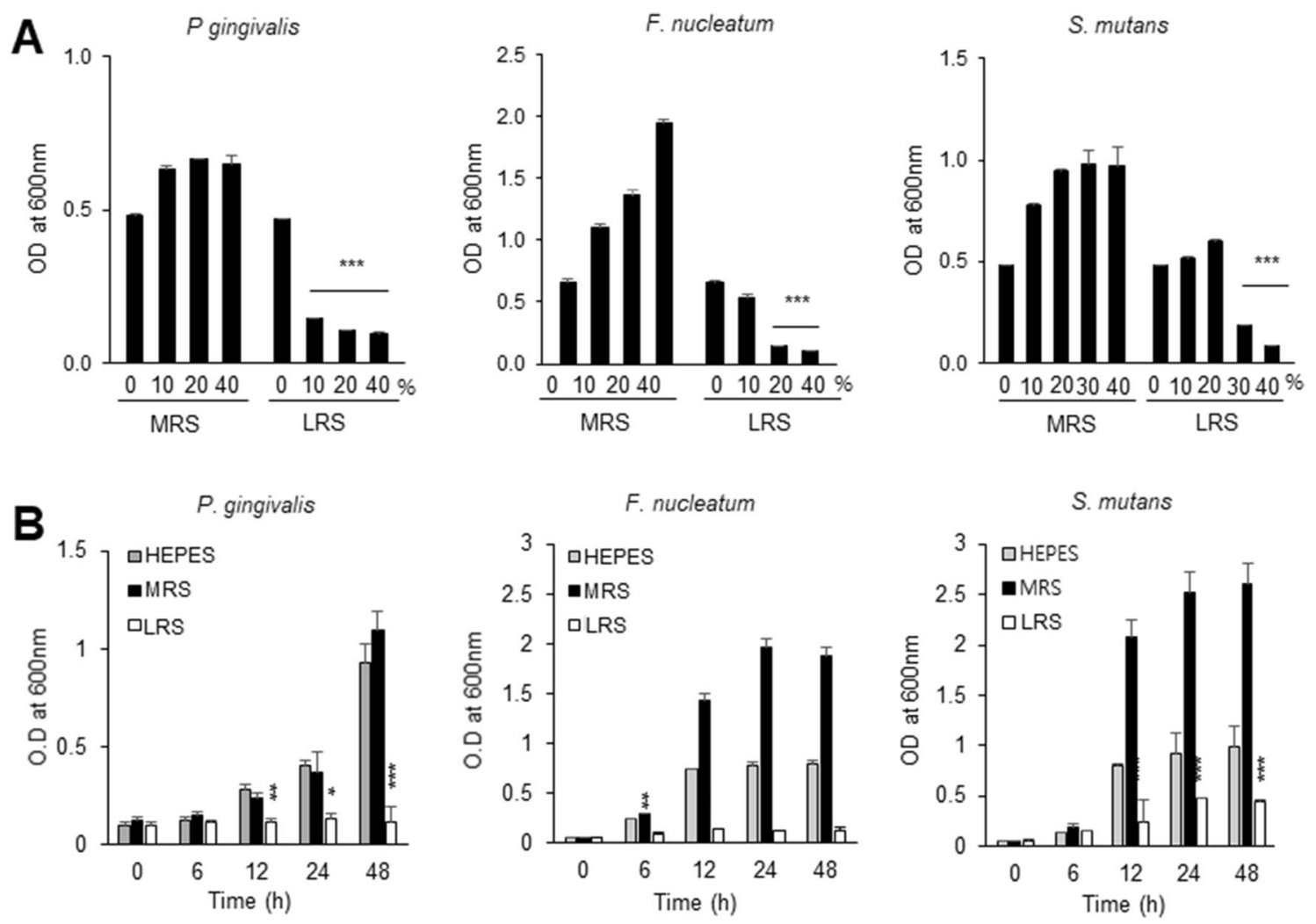

Figure 2. Effect of LRS on the growth of P. gingivalis, F. nucleatum, and S. mutans. (A) The effect of $0 \%(\mathrm{v} / \mathrm{v})$, $10 \%(\mathrm{v} / \mathrm{v}), 20 \%(\mathrm{v} / \mathrm{v})$, and $40 \%(\mathrm{v} / \mathrm{v})$ LRS on the growth of pathogenic oral bacteria. Pure MRS medium was used as the control. Data presented are $\mathrm{OD}_{600}$ values after incubation for $24 \mathrm{~h}$. Significant differences from the control are indicated by $\left.{ }^{(*}\right)$ for $p<0.01$ and $\left(^{* *}\right)$ for $p<0.001$. (B) The effect of $40 \%(\mathrm{v} / \mathrm{v})$ LRS on the growth of pathogenic oral bacteria over time. Pure MRS medium was used as the control. Data presented are culture $\mathrm{OD}_{600}$ values. Significant differences from the control are indicated by $\left(^{\star}\right)$ for $p<0.05$, $\left(^{\star *}\right)$ for $p<0.01$, and $\left({ }^{\star * \star}\right)$ $p<0.001$.

$10 \%, 20 \%$, and $30 \%(\mathrm{v} / \mathrm{v})$ treatment of LRS achieved substantial removal of biofilms (Fig. 4B). Furthermore, we observed a significant reduction in the expression of $\operatorname{rgp} A, \operatorname{rgp} B, \operatorname{hag} A, \operatorname{hagB}$, and $\mathrm{kgp}$, all of which are genes involved in biofilm attachment and formation, in P. gingivalis following LRS treatment (Fig. 4C,D).

Whole genomic sequencing of $L$. reuteri AN417. To determine the antibacterial and antibiofilm molecules produced by $L$. reuteri AN417, whole genome sequencing was performed using PacBio RSII singlemolecule real-time (SMRT) sequencing technology. As shown in Supplementary Fig. S2, the complete genome consisted of a single circular chromosome of 2,069,421 bp and four circular plasmids of 93,397 bp (pLreu417A of 57,676 bp, pLreu417B of 16,368 bp, pLreu417C of 10,268 bp, and pLreu417D of $9085 \mathrm{bp}$ ) with 38.95\% G+C content. A total of 2151 genes were predicted in the genome of this strain. Of these, 2034 were identified as protein-coding genes. The total length of the coding regions was $1,850,478 \mathrm{bp}$. A total of 1571 protein-coding genes were assigned putative functions. The remainder were annotated as hypothetical proteins.

Genomic analysis and characterization of $L$. reuteri AN417. Genome-genome relatedness of $L$. reuteri AN417 was also analyzed by calculating the average nucleotide identity (ANI) and constructing a phylogenomic tree of 31 genome sequences with fewer than 30 scaffolds among the genomes of $L$. reuteri strains from the GenBank/EMBL/DDBJ database. L. reuteri genomes were an average size of $2.10 \mathrm{Mb}$ and $\mathrm{G}+\mathrm{C}$ ratio of 38.43-39.31 (Table 2). The whole genome of L. reuteri AN417 showed ANI values ranging from 94.8 to $99.6 \%$ with $L$. reuteri strains. The highest ANI values were obtained for the pig strains (Fig. 5A). Additionally, whole genome phylogenetic analysis was performed with 27 L. reuteri genomes, excluding those with many contigs. A phylogenetic tree of $L$. reuteri strains was constructed using the amino acid alignments of 766 core genes using the maximum likelihood approach. The tree showed clear separation of L. reuteri strains into five host-defined phylogenetic lineages (Fig. 5B). This analysis also revealed that L. reuteri AN417 isolated from pigs clustered in lineage IV, which is contained in strains originating from pigs.

The whole genome sequence of $L$. reuteri AN417 encodes the urease complex UreABCEFGD unlike general strains isolated from human and pigs, contributing to its viability under acidic conditions (Fig. 5C). The mucin binding protein, Muc2, contributes to host adaptation and adhesion to mucus. However, genes encoding reuteran, reutericyclin, and reuterin, which are important in the antimicrobial activity of $L$. reuteri strains, were absent in 
A
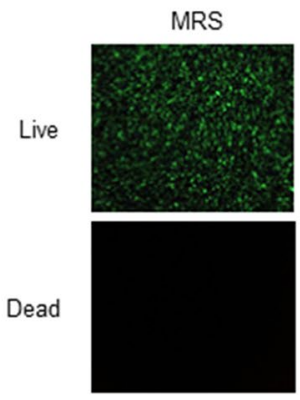

B
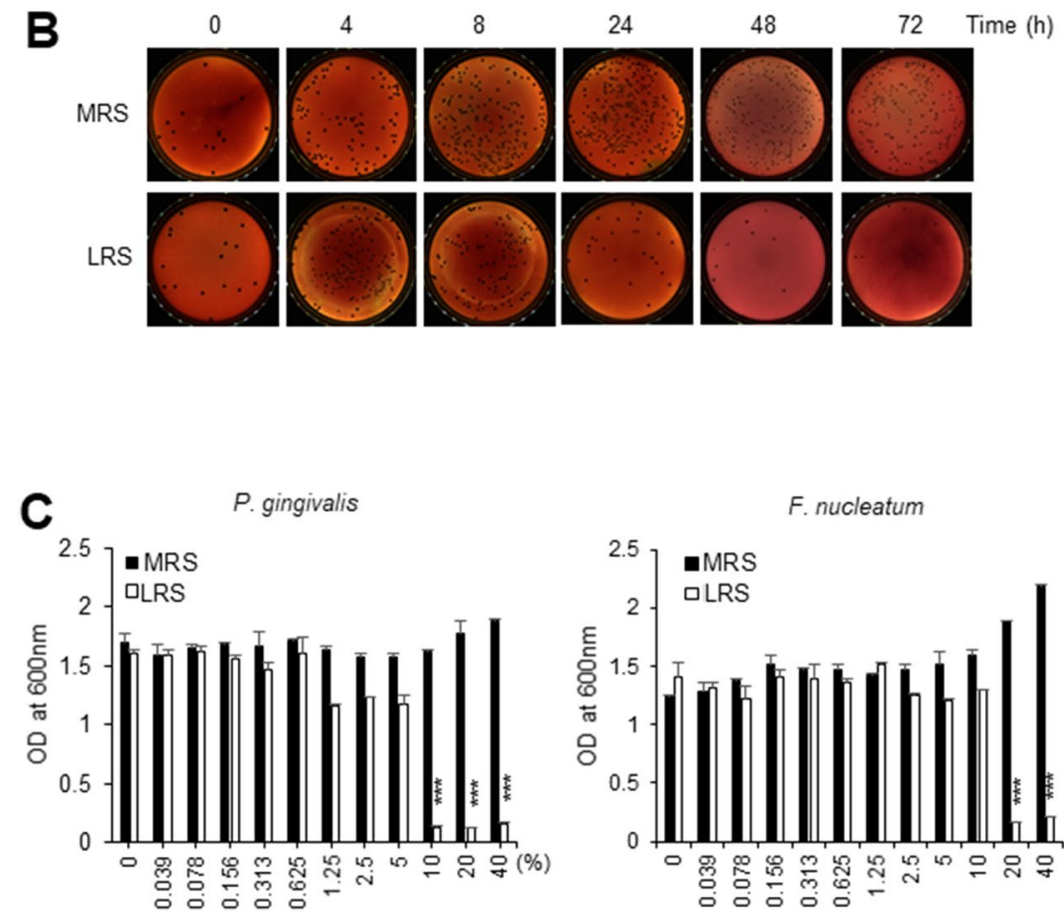

LRS
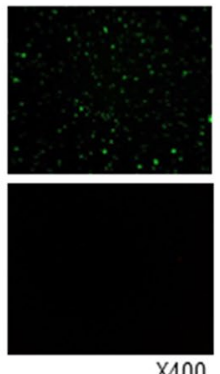

$\mathrm{X} 400$
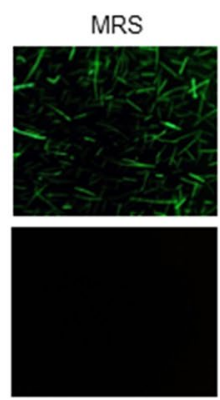

F. nucleatum
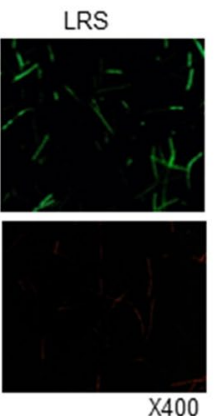

F. nucleatum

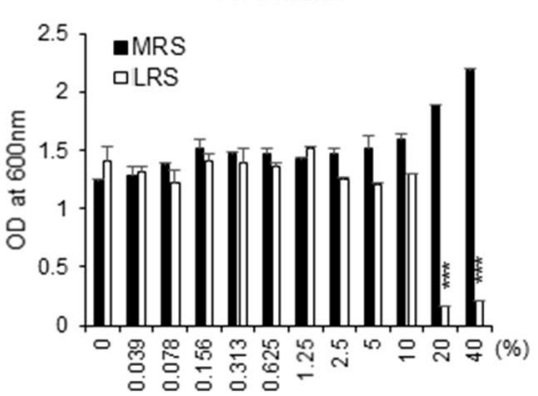

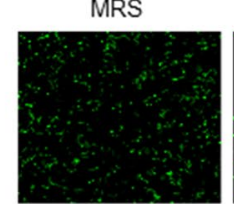
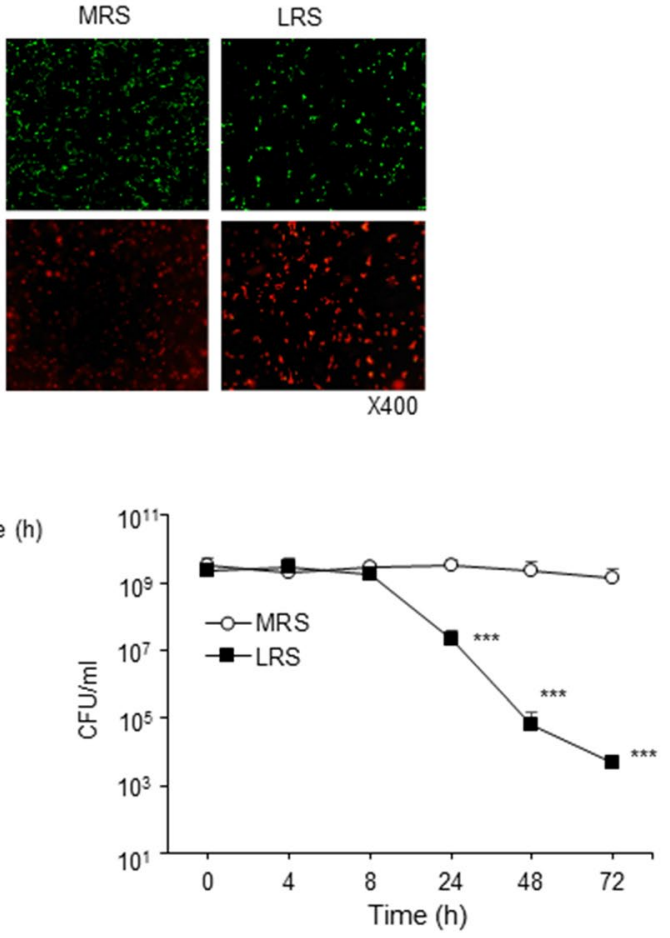

Figure 3. Analysis of the MIV of LRS and the effect of LRS on P. gingivalis, F. nucleatum, and S. mutans cell viability. (A) Representative fluorescence images of LIVE/DEAD BacLight viability assay of pathogenic bacteria exposed to $20 \%$ (v/v) LRS or MRS broth (control) for $24 \mathrm{~h}$ at $37^{\circ} \mathrm{C}$. Red fluorescence indicates dead or membrane-damaged bacterial cells and green fluorescence indicates live/healthy bacteria. Original magnification, $\times 400$. (B) Quantification of $P$. gingivalis cell concentrations $(\mathrm{CFU} / \mathrm{mL}$ ) following treatment with $20 \%(\mathrm{v} / \mathrm{v}) \mathrm{MRS}$ (control) or LRS. Black colonies developed after 6 days growth in a $37^{\circ} \mathrm{C}$ anaerobic chamber. The time required to kill $P$. gingivalis treated with MRS or LRS is shown graphically. Significant differences from the control $(p<0.001)$ are indicated by ${ }^{* *}$. (C) MIV of LRS for pathogenic bacteria, determined based on $\mathrm{OD}_{600}$ values after $48 \mathrm{~h}$ incubation in an aerobic or aerobic chamber maintained at $37^{\circ} \mathrm{C}$. Significant differences from the control $(p<0.001)$ are indicated by ${ }^{* * *}$.

the genome (Fig. 5C). In addition, reuterin production was also measured in L. reuteri AN417 (Table 2), demonstrating that $L$. reuteri AN417 did not produce reuterin. This result was consistent with the genomic analysis results (Table 1).

Antibacterial activity of LRS is mediated by the presence of fatty acids and sugars. To categorize the type of metabolites responsible for the activity of LRS against periodontopathogens, the antibacterial effect of LRS against $P$. gingivalis in the presence of proteinase $\mathrm{K}$, lipase, and $\alpha$-amylase was evaluated. Treatment of LRS with lipase or a-amylase eliminated the inhibitory effect of LRS on P. gingivalis growth (Fig. 6A-C). These findings suggested that the antibacterial effect of LRS against oral pathogenic bacteria could be attributed to the presence of a fatty acid and/or sugar. 
A
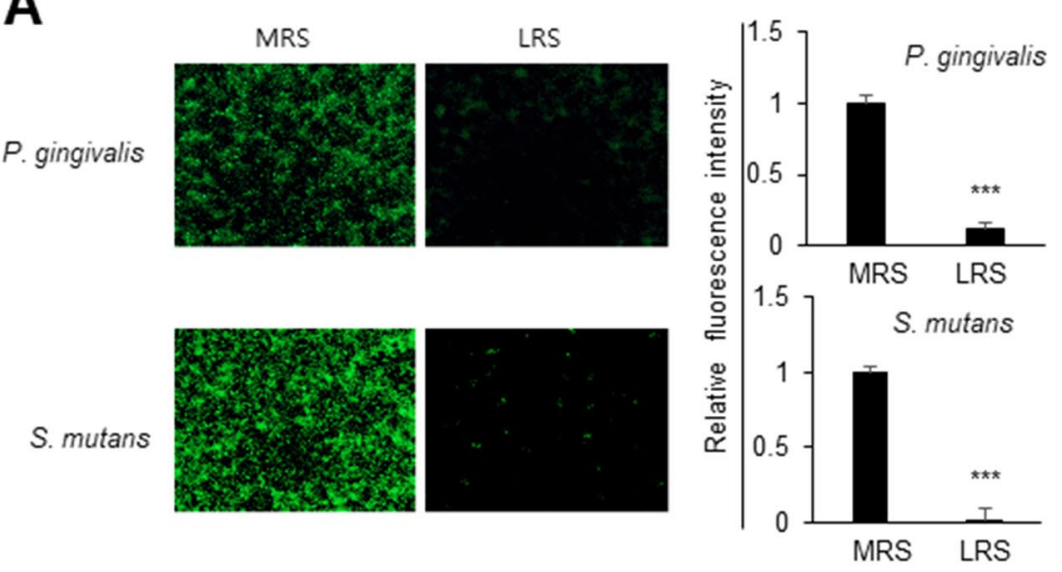

B
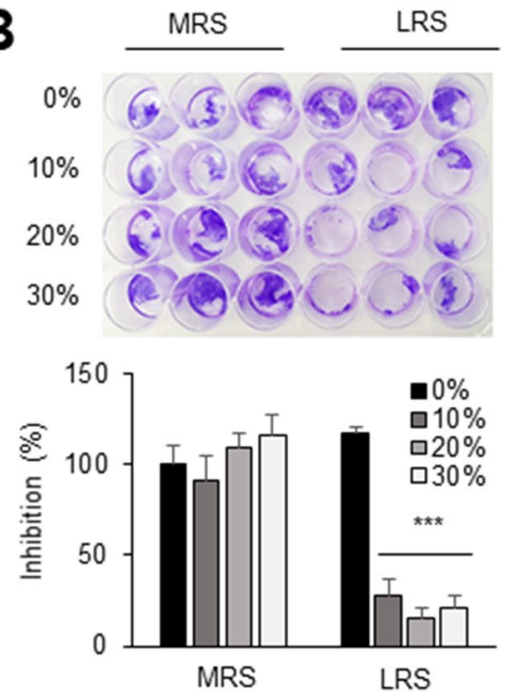
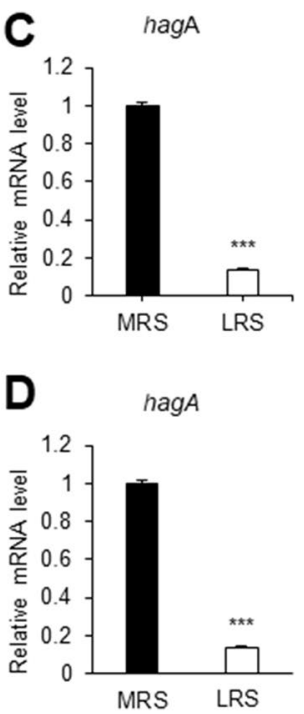

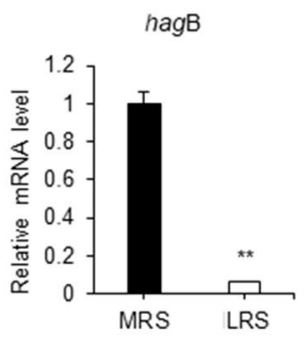

hagB

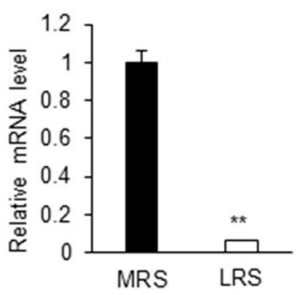

$k g p$

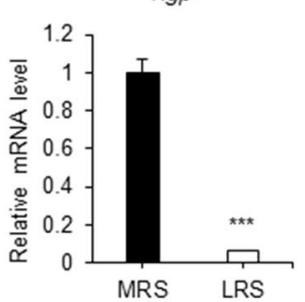

$k g p$

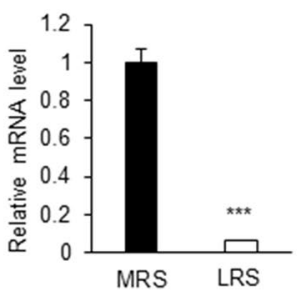

rgpA

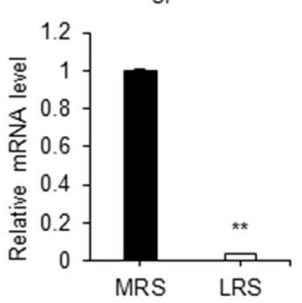

rgpA

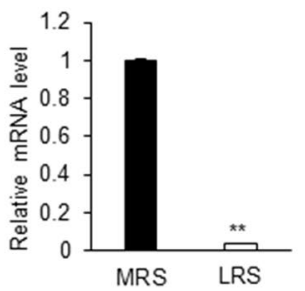

rgpB

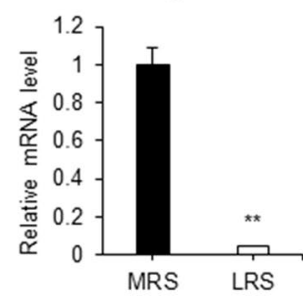

$r g p B$

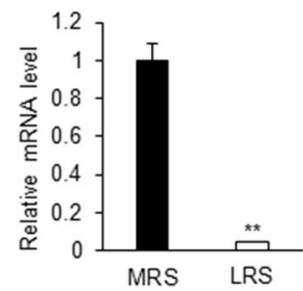

Figure 4. Activity of LRS against $P$. gingivalis and S. mutans biofilm formation (A) Visualization using fluorescence microscopy (left panel) of the antibiofilm effect of LRS. Non-adherent bacteria were removed, and the biofilms stained using the LIVE/DEAD BacLight Bacterial Viability Kit. Green and red fluorescence indicates live and dead bacteria, respectively. The fluorescence intensity ratio of live/dead cells (right panel) was analyzed using ImageJ software (IJ version 1.46r; https://imagej.nih.gov/ij/download.html). Significant differences from the control $(p<0.001)$ are indicated by ${ }^{* * *}$. (B) Images showing crystal violet-stained biofilms of $P$. gingivalis following treatment with various concentrations of MRS (control) and LRS. Significant differences from the control $(p<0.001)$ are indicated by ${ }^{* * *}$. (C,D) The effect of LRS on the expression of genes involved in biofilm formation. Bacteria were treated with MRS broth (control) or LRS for $48 \mathrm{~h}$ following (C) initial biofilm colonization or (D) following established biofilm formation (5 days). The mRNA levels of hagA, hagB, $\operatorname{rgpA}$, $r g p B$, and $k g p$ genes were quantitatively measured using RT-qPCR. The data are expressed as the relative level of 16S rRNA. Significant differences from the control are indicated by $\left({ }^{* *}\right) p<0.01$ and $\left({ }^{* * *}\right) p<0.001$.

\section{Discussion}

The present study reports the antibacterial effects of cell-free culture supernatant from L. reuteri strain AN417 (LRS), a strain that was isolated from the porcine small intestine, against selected oral pathogenic bacteria. Based on our results, we anticipate that $L$. reuteri AN417 can positively affect oral health. Recently, there has been growing interest in the potential and utilization of microbial metabolites, termed postbiotics ${ }^{23}$. The cell-free culture supernatant of $L$. reuteri AN417 exhibited greater antimicrobial activity than those of the known Lactobacillus reference strains KCTC 3594 and KCTC 3678, which also inhibited periodontopathic bacteria (Fig. 1A,B). In our study, multiple lines of scientific evidence validated the antimicrobial activity of LRS.

Evaluation of the antimicrobial effects of naturally-derived agents has largely focused on their activity against P. gingivalis, F. nucleatum, and S. mutans because these bacteria have received the most attention in relation to oral diseases and are implicated in periodontal diseases, dental caries, and endocarditis ${ }^{7-10}$. To date, the antimicrobial effects of naturally derived agents against these bacteria have been evaluated in numerous studies, with 


\begin{tabular}{|c|c|c|c|c|c|c|c|c|}
\hline Species & Strains & $\begin{array}{l}\text { Genome assembly } \\
\text { acc. No }\end{array}$ & Countries & Origin & No. of contigs & Genome size (bp) & GC ratio (\%mol) & Notes \\
\hline L. reuteri & 121 & GCA_001889975.1 & Netherlands & Pig & 14 & $2,302,234$ & 39.01 & - \\
\hline L. reuteri & 2010 & GCA_003703885.1 & USA & Rat & 38 & $2,220,255$ & 38.53 & - \\
\hline L. reuteri & $100-23$ & GCA_000168255.1 & New Zealand & Rat & 2 & $2,305,557$ & 38.73 & - \\
\hline L. reuteri & ATCC 53,608 & GCA_000236455.2 & Sweden & Pig & 3 & $2,091,243$ & 38.75 & - \\
\hline L. reuteri & ATG-F4 & GCA_004208615.1 & Korea & Human & 1 & $2,041,516$ & 38.89 & - \\
\hline L. reuteri & Byun-re-01 & GCA_003316895.1 & Korea & Mouse & 1 & $2,244,514$ & 38.88 & - \\
\hline L. reuteri & CNI-KCA2 & GCA_012275185.1 & Nigeria & Chicken & 1 & $2,072,001$ & 38.92 & - \\
\hline L. reuteri & CRL 1098 & GCA_001657495.1 & Germany & Sourdough & 45 & $1,963,029$ & 38.74 & - \\
\hline L. reuteri & DSM 20,016 & GCA_000016825.1 & - & Human & 1 & $1,999,618$ & 38.87 & Type strain \\
\hline L. reuteri & I49 & GCA_001688685.2 & Switzerland & Mouse & 1 & $2,044,771$ & 38.76 & - \\
\hline L. reuteri & I5007 & GCA_000410995.1 & China & Pig & 7 & $2,093,275$ & 38.93 & - \\
\hline L. reuteri & IRT & GCA_001046835.1 & Korea & Human & 1 & \begin{tabular}{|l|}
$1,993,967$ \\
\end{tabular} & 38.90 & - \\
\hline L. reuteri & L6798 & GCA_900093565.1 & Sweden & Mouse & 35 & $2,108,374$ & 38.43 & - \\
\hline L. reuteri & LL7 & GCA_007633215.1 & USA & Mouse & 2 & $2,384,717$ & 38.81 & - \\
\hline L. reuteri & \begin{tabular}{|l|} 
LTH2584 \\
\end{tabular} & GCA_000712555.1 & Germany & Sourdough & 55 & $2,066,054$ & 38.53 & - \\
\hline L. reuteri & \begin{tabular}{|l|} 
LTH5448 \\
\end{tabular} & GCA_000758185.1 & Germany & Sourdough & 36 & $1,980,298$ & 38.44 & - \\
\hline L. reuteri & \begin{tabular}{|l|} 
LTR1318 \\
\end{tabular} & GCA_009184725.1 & China & Human & 2 & $2,047,619$ & 38.98 & - \\
\hline L. reuteri & Marseille-P4870 & GCA_901600665.1 & France & Yogurt & 287 & $2,039,591$ & 38.98 & $\begin{array}{l}\text { For only ANI } \\
\text { calculation }\end{array}$ \\
\hline L. reuteri & Marseille-P4904 & GCA_901600705.1 & France & Yogurt & 282 & $2,039,591$ & 38.98 & $\begin{array}{l}\text { For only ANI } \\
\text { calculation }\end{array}$ \\
\hline L. reuteri & Marseille-P5460 & GCA_901600695.1 & France & Yogurt & 316 & $2,039,540$ & 39.21 & $\begin{array}{l}\text { For only ANI } \\
\text { calculation }\end{array}$ \\
\hline L. reuteri & Marseille-P5461 & GCA_901600675.1 & France & Yogurt & 311 & $2,039,572$ & 39.21 & $\begin{array}{l}\text { For only ANI } \\
\text { calculation }\end{array}$ \\
\hline L. reuteri & MM4-1A & GCA_000159475.2 & Finland & Human & 7 & $2,067,914$ & 38.88 & - \\
\hline L. reuteri & SD2112 & GCA_000159455.2 & Peru & Human & 5 & $2,316,838$ & 39.04 & - \\
\hline L. reuteri & \begin{tabular}{|l|} 
SKKU- \\
OGDONS-01
\end{tabular} & GCA_003316935.1 & Korea & Chicken & 1 & $2,259,968$ & 38.86 & - \\
\hline L. reuteri & TD1 & GCA_000439275.1 & USA & Rat & 1 & $2,145,445$ & 38.78 & - \\
\hline L. reuteri & TMW1.112 & GCA_000722535.2 & Germany & Sourdough & 12 & $2,032,034$ & 38.45 & - \\
\hline L. reuteri & TMW1.656 & GCA_000712565.2 & Germany & Sourdough & 17 & $1,949,539$ & 38.49 & - \\
\hline L. reuteri & UBLRU-87 & GCA_003719715.1 & India & Fermented food & 91 & $1,821,307$ & 38.70 & $\begin{array}{l}\text { For only ANI } \\
\text { calculation }\end{array}$ \\
\hline L. reuteri & WHH1689 & GCA_003072625.1 & China & $\begin{array}{l}\text { Highland barley } \\
\text { wine }\end{array}$ & 1 & $2,044,184$ & 39.31 & - \\
\hline L. reuteri & YSJL-12 & GCA_006874665.1 & China & Pig & 3 & $2,151,788$ & 38.93 & - \\
\hline L. reuteri & ZLR003 & GCA_001618905.1 & China & Pig & 1 & $2,234,097$ & 38.66 & - \\
\hline L. reuteri & AN417 & In this study & Korea & Pig & 5 & $2,162,818$ & 38.95 & - \\
\hline L. gastricus & LG045 & GCA_009648555.1 & Korea & Human & 3 & $1,905,155$ & 41.65 & Outgroup for tree \\
\hline L. secaliphilus & DSM 17,896 & GCA_001437055.1 & Germany & Sourdough & 15 & $1,646,143$ & 47.72 & Outgroup for tree \\
\hline
\end{tabular}

Table 2. Lactobacillus reuteri genomes used for phylogeny reconstruction and comparative genomics. Whole genome phylogenetic analysis was performed with 27 L. reuteri genomes except for them with large numbers of contigs.

a recent study reporting the antimicrobial activities of $L$. reuteri supernatant against $P$. gingivalis ${ }^{21}$. Hence, we also focused on these three oral pathogens in our study.

The major antibacterial compounds produced by $L$. reuteri strains are reuterin, reuteran, and reutericyclin. Genomic analysis of $L$. reuteri AN417 revealed the absence of a $p d u$-cbi-cob-hem gene cluster for the biosynthesis of reuterin and cobalamin (vitamin $\mathrm{B}_{12}$ ), and genes for the synthesis of reutericycin and reuteran. However, L. reuteri AN417 encoded an inulin-type fructansucrase. The strain also encoded the UreABCEFGD urease complex, which was not found in the other strains isolated from humans and pigs, contributing to its viability under acidic conditions.

We compared the activity of concentrated L. reuteri AN417 cell-free culture medium (LRS) to the activity of $L$. reuteri AN417 cell extracts using organic solvents. The results showed that $L$. reuteri AN417 cell extracts had no antimicrobial activity, whereas LRS did (Fig. 1C). From these data, secondary metabolites produced during growth are thought to play a crucial role in antimicrobial activities against oral pathogenic bacteria ${ }^{24}$. Bungenstock et al. reported that the antibacterial effect of probiotics against fermented foodborne pathogens is attributable to their production of lactic acid and the associated increase in acidity ${ }^{25}$. However, the antimicrobial activity of LRS observed in this study may not have been due to lactic acid alone. Probiotic Lactobacillus spp. are 
A

\begin{tabular}{|c|c|c|c|c|c|c|c|c|c|c|c|c|c|c|c|c|c|c|c|c|c|c|c|c|c|c|c|c|c|c|c|c|}
\hline & & 1 & 2 & 3 & 4 & 5 & 6 & 7 & 8 & & 10 & 11 & 12 & 13 & & 15 & 16 & 8 & 19 & 20 & 21 & 22 & 23 & 24 & 25 & 27 & 28 & & & & & \\
\hline 1 & ATG-F4 & $\cdots$ & 100 & 100 & 100 & 100 & 100 & 9 & 95.49 & 95.79 & 96.29 & 96.196 & 96.196 & 96.196 & 96.196 & 96.296 .4 & $\begin{array}{ll}6.495 .8 \\
\end{array}$ & 895.89 & 95.79 & 96.49 & 96.59 & 96.59 & (96.1 & 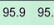 & $\begin{array}{ll}95.9 & 95 .\end{array}$ & $\begin{array}{l}.996 .6 \\
\end{array}$ & 96.69 & & 96.496. & 6.395. & 9 & \\
\hline 2 & DSM & & $\cdots$ & 100 & 100 & 100 & 100 & 9989 & 95.39 & 95.79 & 96.296 & 9629 & 96.196 & 96.1 & $\begin{array}{ll}96 & 96\end{array}$ & 96.296 .3 & $\begin{array}{ll}6.3 & 95.7\end{array}$ & 795.79 & 95.79 & 96.49 & 96.59 & 96.5 & 9699 & 95.8 & 5.995. & 6.6 & 9 & 96 & 9 & 0.59 & & \\
\hline 3 & IRT & & 99.9 & $\ldots$ & 100 & 99.99 & 99.99 & 99.89 & 95.39 & 95.89 & 96.196 & 96.196 & 96.196 & 96.196 & 96.196 & 96.296 .4 & 6.495 .8 & 895.7 & 95.79 & 96.49 & 96.59 & 96.59 & 96.195 & 95.995 & 9 & 6.7 & 69 & 96.496 & 96 & 95. & 5.9 & \\
\hline 4 & $\mathrm{JCI}$ & 00 & 100 & 100 & $\cdots$ & 100 & 1009 & 99.89 & 95.49 & 95.79 & 96.296 & 96.196 & 96.196 & 96.196 & 96.196 & 96.296 .4 & 6.495 .8 & 895.8 & 95.79 & 96.49 & 96.5 & 96.59 & 96.19 & 95.995. & 95. & 6 & 9 & 96.496 & 9 & 95. & 5.9 & \\
\hline 5 & MM4-1 & 100 & 100 & 100 & 100 & -- & 100 & 9989 & 95.49 & 95.79 & 96.296 & 96.196 & 96.196 & 96.196 & 96.196 & 96.296 .4 & 6.495 .8 & 895.8 & 95.79 & 96.49 & 96.59 & 96.59 & 96.195 & 95.995 . & 55995 & 6 & 9 & 96.496 & 96.496. & 6.395. & 5.9 & \\
\hline 6 & CRL 1 & .9 & 100 & 99.9 & 99.9 & 99.9 & -- & & 95.39 & 95.89 & 96.296 & 96.296 & 96.196 & 96.19 & $96 \quad 96$ & 96.196 .4 & 6.495 .8 & 895.7 & 95.79 & 96.49 & 96.6 & 96.5 & 969 & 95.895. & 95. & 6 & 5 & 96.496 & 96.496. & 95. & 5.9 & \\
\hline 7 & LTR13 & 0.8 & 99.8 & 99.8 & $99.8 \quad 9$ & 99.89 & 99.8 & $-{ }_{-1}$ & 95.39 & 95.99 & 96.296 & 96.196 & 96.196 & 96.196 & 96.196 & 96.396 .4 & 6.495 .7 & 95.79 & 95.79 & 96.39 & 96.59 & 96.59 & 96.19 & 95.99 & 95. & 6.7 & 9 & 96.496 & $96.4 \quad 96$. & 6.2 & 5995 & \\
\hline 8 & SD21 & 95.59 & 95.49 & 95.49 & 95.49 & 95.49 & 95.49 & 95.5 & --9 & 9869 & 9889 & 98798 & 98898 & 98.895 & 95.195 & 95.395 .4 & 5.495 .4 & 495.1 & 95.39 & 95.3 & 969 & 95.99 & 95.19 & 94.99 & 35995 & 3 & 99 & 95 & 95.595. & 5.294. & 4.994. & \\
\hline 9 & CNI-K & 9 & 95.89 & 95.9 & 95.89 & 95.79 & 95.79 & 95.99 & 98.3 & --9 & 98.798 & 98698 & 98798 & $\begin{array}{ll}98.7 & \mathrm{~g}\end{array}$ & 959 & 9595. & 5.495 .2 & 294.8 & 95 & 95 & 95.9 & 95.79 & 94.89 & 94.7 & 95 & 3 & 95.99 & $\begin{array}{l}95.8 \quad 95 \\
\end{array}$ & 95.3 & 95 & 4.694 & \\
\hline N & $M a$ & 96 & 96.19 & 96.1 & 96 . & 96 & 96 & $96.1 \quad 9$ & 98.59 & 98.6 & -- & $99.9 \quad 1$ & 100 & 10095 & 95.395 & $95.3 \quad 95.4$ & 5095.4 & $495.3=$ & 95.49 & 95.59 & 96.29 & $96.2 \mathrm{~g}$ & 95 & 95 & 35.6 & & 96.3 & 96 & 95. & 5.4 & 35 & \\
\hline n & & 9 & 96.19 & 96 & 96.1 & 96 & 96 & 96.1 & 8.59 & $98.6 \quad 1$ & 100 & --1 & 100 & 10095 & 95.395 & 95.395 .4 & $\begin{array}{ll}5.4 & 95.3\end{array}$ & 395.39 & 95.4 & 95.59 & 96.29 & 96.19 & 95 & 95 & & & 96.2 & 96 & 96.195. & 5.4 & 55 & \\
\hline$n$ & M & & 96 & & 96 & 95.9 & 96 & 95.99 & 8.49 & 98.69 & 99.99 & 99.9 & -- & 95 & 95.395 & 95.295. & 5.495 .4 & 495.3 & 95.3 & 95.4 & 96.2 & 96 & 95 & 95 & 35. & & 96.2 & 36 & 95. & 5.3 & 95 & \\
\hline N & & & 96 & 95 & 96 & 95 & 96 & 5.9 & $98.4 \quad 98$ & 98.69 & 99.999 & 99.9 & 100 & $\ldots-95$ & 95.395 & $95.2 \quad 95.4$ & 5.495 .4 & 39 & 95.39 & 95.49 & 96 & 969 & 95 & 95 & & & 96.2 & 36 & 35. & 5.3 & & \\
\hline $4 \mathrm{~A}$ & & & & & 96.49 & 96 & 96.29 & & 95 & 3 & 695 & 595 & 95.595 & 95.5 & $\begin{array}{c}\ldots-99 \\
-1\end{array}$ & 99.199 .6 & 9.699 .4 & 4 & 99 & $\begin{array}{ll}99.2 \\
9\end{array}$ & & 95.1 & & 96.1 & & & & & & 95 & & \\
\hline & & & & & & & 96.2 & & & & & & & & 99.1. & $\cdots$ & 9.3 & & t9.3 & ty.1 & & 95.79 & & 96.195. & 35. & & & 95.795 & 95.296. & b. & & \\
\hline & & & & & & & & & & & & & & & $99.6 \quad 99$ & $99.3 \quad \ldots$ & 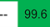 & & & 99.39 & 95.69 & & & & & & & & 296. & 6.3 & & \\
\hline & & & & & & & & & & & & & & & & 99.299 .6 & 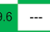 & & & 99.2 & . & & & 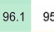 & & & & 95 & S.. & 0.2 & & \\
\hline & & & & & & & & & & & & & & & & 99 & 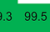 & $\cdots$ & & & 95.4 & 95.39 & 95.9 & 95.994 & 4.9 & & & . & $\begin{array}{ll}95 & 96 .\end{array}$ & 6.1 & 95. & \\
\hline 2 & & & & & & & & & & & & & & & & 99.3 & 9.4 & & & & & 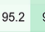 & 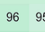 & 95.8 & & & & 94 & 94 & 96 & 96 & \\
\hline 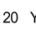 & & & & & & & & & & & & & & & 99.1 & $99 \quad 99.3$ & 9.399 .2 & 298.7 & 98.9 & $-\cdots$ & 69 & 95.6 & 9 & 36 & 95. & 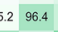 & 9 & 95 & 95. & 96. & 6.1 & \\
\hline & & & & & & & & & & & & & & & 95.395 & 95.6 & 5.7 & 39 & $95.4 \mathrm{~g}$ & 95.7 & $\cdots$ & & 95.39 & 95.4 & 96. & 95.7 & 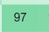 & 9 & 97 & 95. & 94. & \\
\hline 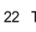 & 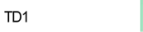 & & 96.59 & 96 & 96 & & 96 & 96. & & & 396 & & & 94 & 94.995 & 95.5 & 5.7 & 19 & 95.19 & 95.6 & 9) & $\ldots$ & 95.599 & 95.3 & 96. & 5 & 96. & 96 & 96 . & 5.7 & 5.4 & \\
\hline & & & 96 & 50.0 & 9 & & 96 & & & & & & & & 95.896 & 96.196 .3 & 6.3 & 65 & 9 & 96 & 9 & 95. & $\ldots g \varepsilon$ & 98.2 & 94. & 95.4 & 95.7 & 9 & 97. & 7.3 & 7.8 & \\
\hline 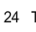 & & & 95.9 & 96 & 9 & & 9 & & & & 2 & 195 & 2 & 9 & $96 \quad 96$ & 96.196 .3 & 6.3 & 295.99 & 95.89 & 96.19 & 9 & 39 & 98.3 . & $\cdots$ & 95. & 5.3 & 95.5 & 95.6 & 95 & 7.3 & 95. & \\
\hline$s$ & & & 90 & 96 & & & & & & & 995 & 95.995 & 95.99 & & 95.195 & 5.5 & 5.5 & 959 & & 95.5 & 9 & 96.3 & 9 & $95.1 \quad \ldots$ & 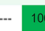 & 3 & 96.49 & 95 & 95.9 & 5. & 94. & \\
\hline E & B & & 9 & & & & 96 & 9 & & & 95.995 & & 5.9 & 99 & 95.195 & 95.4 & .5 & 4 & 29 & 95.59 & 9 & 96.4 & & $\begin{array}{l}95.1 \\
10\end{array}$ & 100 & , & 96.4 & 96.3 & 95. & 94. & 94. & \\
\hline & & & & & & & & & & & 79 & 95.795 & 395 & 79 & 96 & 6.5 & 5.8 & 49 & & & 95 & 9 & & 95. & 55.395. & 5.3 & 95.9 & 95 & & 95. & 95. & \\
\hline & 14 & & & & & & & & & & & 96 & & & 95 & 95.795. & 9 & 5.3 & & 8 & 9 & 96.7 & & & 96 & 3 & $\ldots 9$ & 96.896 & 95. & 5.6 & 94. & \\
\hline & & & & & & & & & & & & 96 & & & 95 & 95 & 8 & 28 & & & & 96.89 & & & 35. & 96 & & 96 & 95. & 5. & & \\
\hline & & & & 96 & & & & & & & & & & & 94.995 & 95.195 .5 & 15 & 95 & $94.9 \mathrm{~g}$ & & & 97 & & & 96 & 5 & 9 & 97 & 95. & 5.595. & & \\
\hline & & & & 96 & & & & & & & & 95.4 & & & $\begin{array}{ll}95.9 & 96\end{array}$ & $96.1 \mathrm{~g}$ & & 96 & 96 & & & & 97 & & & & & & & $\begin{array}{ll}\ldots .- & 97\end{array}$ & 7. & \\
\hline & & & & & & & & & & 1.699 & 95 & 95 & & & 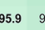 & 96 & & 89 & 95.99 & & 9 & 95.4 & 98 & & 94. & 4.89 & & & & 7. & & \\
\hline & & & & & & & & & & & 9 & 94.8 & & & 95.895 & 95.7 & & & & & & & & & & & & & & & & \\
\hline
\end{tabular}

B

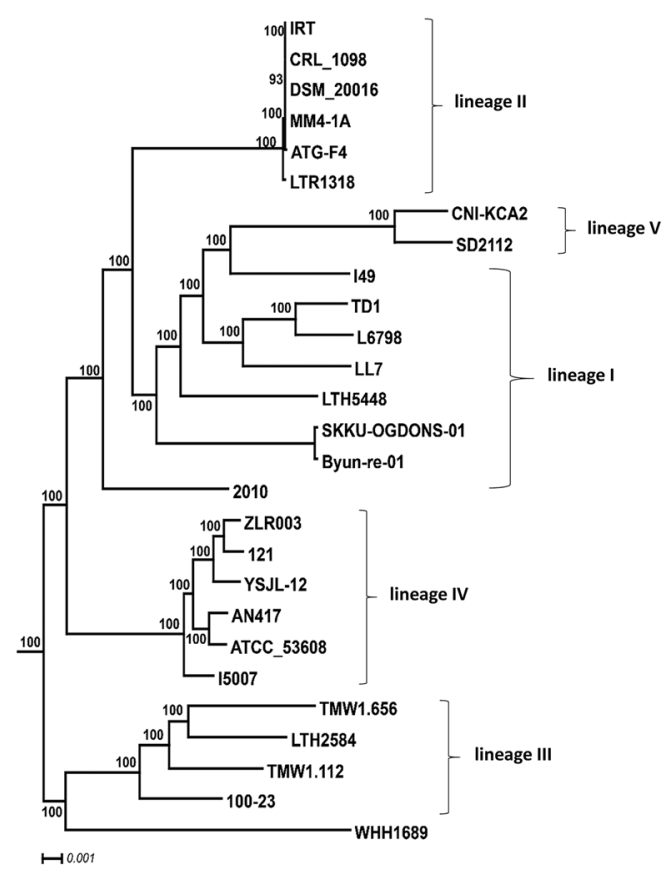

C

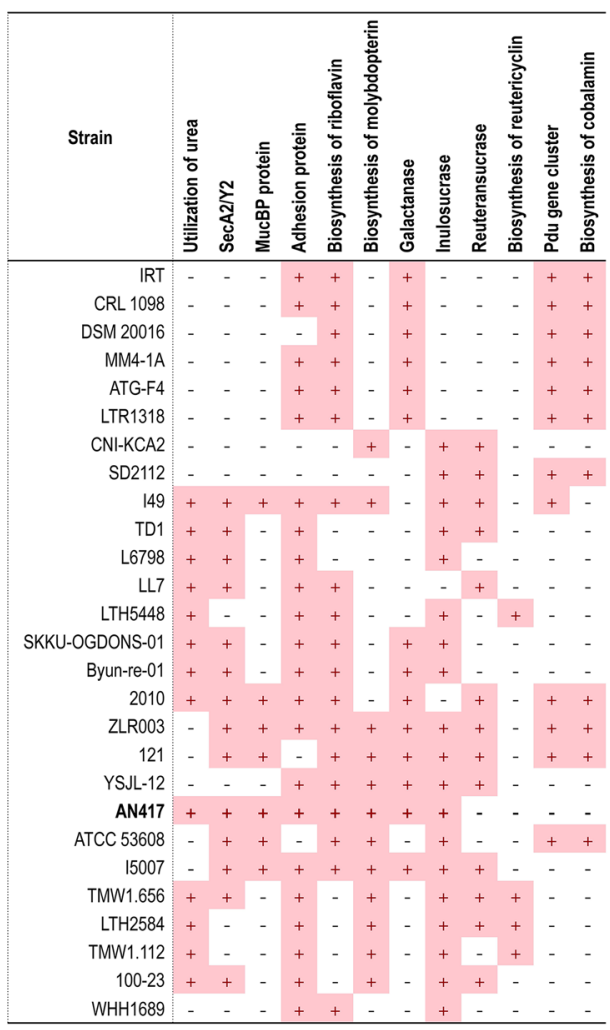

Figure 5. ANI values between genomes of $L$. reuteri strains and their phylogenetic position. (A) ANI values between genomes of $L$. reuteri strains. (B) Phylogenetic tree reconstructed using the amino acid alignments of 766 core genes using the maximum likelihood approach. Numbers above branches show maximum likelihood bootstrap supports from 500 non-parametric replicates. The tree was rooted using L. gastricus LG045 and L. secaliphilus DSM 17896 as outgroups. The scale bar represents the number of substitutions per site. (C) Genes encoding proteins related to environmental adaptation and antimicrobial compounds. 

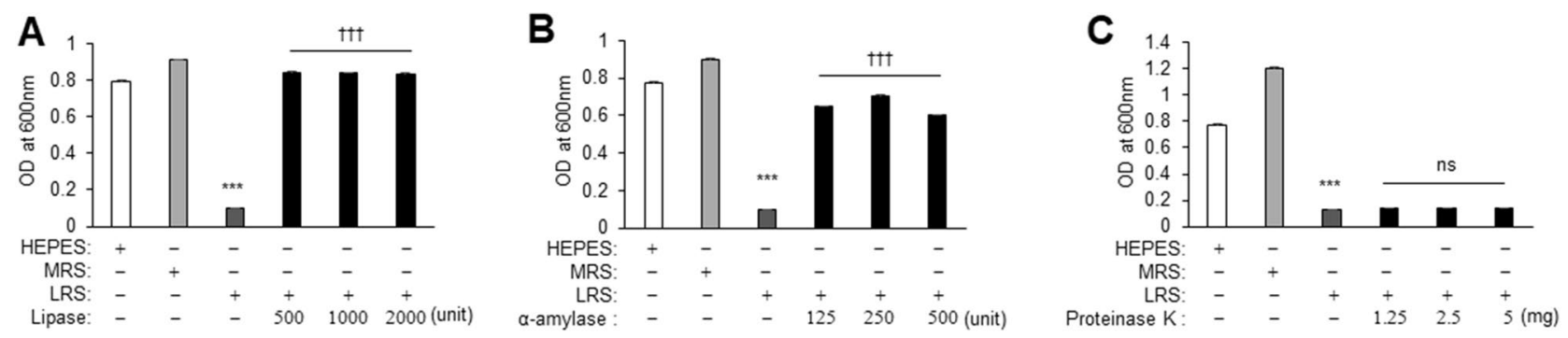

Figure 6. Exploring antibacterial substances in LRS. (A-C) Antibacterial effects of LRS and enzyme-treated ( $\alpha$-amylase, lipase, and proteinase) LRS against $P$. gingivalis after a $24 \mathrm{~h}$ incubation. Significant differences from control and LRS treatments $(p<0.001)$ are indicated by $\left({ }^{* * *}\right)$ and $\left({ }^{\dagger \dagger \dagger}\right)$, respectively.

also known to produce various metabolites that defend against $S$. mutans colonization ${ }^{26}$. The level of intracellular ATP, the energy source for viable oral pathogenic bacteria, was reduced significantly by LRS, leading to the growth inhibition of oral pathogenic bacteria (Fig. 1D, right). The inhibitory effect was maintained for $96 \mathrm{~h}$, indicating that the bioactive substances in the LRS were stable over time. Growth of S. mutans (a Gram-positive bacterium), F. nucleatum (Gram-negative), and P. gingivalis (Gram-negative) was inhibited by adding $30 \%(\mathrm{v} / \mathrm{v})$, $20 \%(\mathrm{v} / \mathrm{v})$, and $10 \%(\mathrm{v} / \mathrm{v})$ of LRS, respectively (Fig. 2A), demonstrating that bacterial growth inhibition by LPS was the most potent and most specific for P. gingivalis. In addition, we speculate that higher concentrations of antimicrobial substances are required to inhibit the growth of Gram-positive bacteria, which have thick peptidoglycan cell walls.

Endotoxins, such as lipopolysaccharides (LPS), are produced from oral bacterial biofilms, including oral plaques. Endotoxins destroy alveolar bone and induce a series of inflammatory reactions that ultimately lead to tooth $\operatorname{loss}^{27}$. To determine whether the effect originated from bacterial membrane damage, SYTO9 and propidium iodide staining was performed. LRS treatment resulted in a marked decrease in viable bacterial cells (Fig. 3). This result may imply that bacterial membrane integrity was weakened by treatment with LRS, which led to the inhibition of bacterial growth and a reduction in the continuous release of endotoxins.

Biofilms are the cause of dental bacterial infections ${ }^{28,29}$. Biofilms are comprised of extracellular polymeric substances secreted by bacteria during metabolic processes. The biofilm structure confers antibiotic resistance ${ }^{30}$. Biofilms in the oral cavity generally contribute to periodontitis. Within biofilms, resident microbes are resistant to external attacks that include antibacterial agents, and the bacteria dispersed from the biofilms can cause infection ${ }^{31}$. Biofilm dispersal agents might be the most suitable targets for the prevention of periodontitis and dental caries. In previous studies, supernatants from cultures of Lactobacillus sp. inhibited biofilm formation and reduced the expression of genes related to the production of exopolysaccharides ${ }^{26}$. In the current study, LRS significantly reduced biofilm formation (Fig. 4), which could contribute to the prevention of dental caries and periodontitis.

LRS has excellent inhibitory abilities against the growth and biofilm formation of the tested bacteria. In particular, $P$. gingivalis required the lowest amounts of LRS to inhibit its growth and biofilm formation among the bacteria tested in our study. Thus, we focused on P. gingivalis in our other analyses, as this was the pathogen most strongly affected by LRS.

The antimicrobial activity of $L$. reuteri has been attributed to its production of organic acids, hydrogen peroxide, and bacteriocin-like compounds ${ }^{32}$, such as reuterin, reuteran, and reutericyclin ${ }^{33}$. However, L. reuteri AN417 lacks the ability to produce them because of the absence of the required genes. Thus, to identify the antimicrobial substance in LRS, various enzymes such as a-amylase, lipase, and proteinase were added to LRS to catabolize and inactivate any sugars, lipids, or proteins that could confer antimicrobial activity. To confirm which enzymes cause loss of activity, each LRS treated with enzymes was added to P. gingivalis cultures. LRS treated with lipase and $\alpha$-amylase did not inhibit $P$. gingivalis growth, suggesting that the active substance responsible for LRS antibacterial activity was either a fatty acid or a sugar. Although $L$. reuteri reportedly produces antimicrobial molecules, including lactic acid, acetic acid, ethanol, and reutericyclin ${ }^{34}$, fatty acid and sugar-based antimicrobial substances have not yet been reported.

According to a previous study ${ }^{35}$, soluble or immobilized PLNC8 $\alpha \beta$ bacteriocins from L. plantarum strains NC8 and 44048 prevent $P$. gingivalis colonization and pathogenicity. Therefore, it is necessary to conduct additional experiments under various conditions to support the findings of this study. Furthermore, the bioactive substances in LRS should be identified and purified for practical use.

In this study, we show that supernatants derived from L. reuteri AN417 cultures are able to suppress the growth and biofilm formation of oral pathogenic bacteria. Interestingly, L. reuteri AN417 does not produce reuteran, reutericyclin, and reuterin, which are important in the antimicrobial activity of reported $L$. reuteri strains. Although further studies are required, the antibacterial substance in LRS is suspected to be a fatty acid or a sugar, which has not yet been reported. Thus, LRS has potential as a novel bioactive substance for the prevention and treatment of pathogens associated with periodontitis. 


\section{Methods}

Strain isolation and identification. Novel Lactobacillus reuteri strains were isolated from seven infants aged 3-7 years and from the small intestines of 13 6-month-old female pigs in the Republic of Korea. The pigs were fed a mixed diet. The intestinal contents from each child or pig were resuspended and serially diluted in sterile $0.85 \% \mathrm{NaCl}$. Aliquots were cultured anaerobically on MRS agar in an atmosphere of $\mathrm{CO}_{2}: \mathrm{H}_{2}: \mathrm{N}_{2}(7: 7: 86)$ atmosphere. After 2-3 days, single colonies were subcultured on fresh MRS agar. All colonies were selected irrespective of their shape and size. Genomic DNA (gDNA) was extracted and purified from cells grown on MRS agar as described previously ${ }^{36}$. The gDNA was used for $16 \mathrm{~S}$ rRNA gene amplification and sequencing, and whole genome sequencing. The complete $16 \mathrm{~S}$ rRNA gene sequence was amplified using universal primers: $27 \mathrm{~F}$ (5'-AGAGTTTGATCMTGGCTCAG-3') and 1492R (5'-TACGGYTACCTTGTTACGACTT-3'). The amplified genes were sequenced and compared with sequences obtained from the EzBioCloud ${ }^{37}$ and GenBank/EMBL/ DDBJ (http://www.ncbi.nlm.nih.gov/blast) databases.

Identification of isolates using a MALDI-TOF Biotyper. One colony of each bacterial isolate was subcultured for $24 \mathrm{~h}$ and used for MALDI-TOF Biotyper analysis. The colony was acquired using a toothpick and spotted onto a polished steel MALDI target plate. One microliter of formic acid ( $70 \%$ in water) was added to the spot and dried. Subsequently, $1 \mu \mathrm{L}$ of MALDI matrix $(10 \mathrm{mg} / \mathrm{mL}$ solution of $\alpha$-cyano-4-hydroxycinnamic acid (HCCA) in $50 \%$ acetonitrile/2.5\% trifluoroacetic acid) was added to the spot and dried. The MALDI target plate was placed in the MALDI-TOF/Microflex LT instrument (Bruker Daltonics, Billerica, MA, USA) for automated measurement and data interpretation. The MALDI Biotyper output is a log (score) between 0 and 3.0, which is calculated from a comparison of the peak list from an unknown isolate with the reference MSP in the database. A $\log$ (score) $\geq 1.7$ was indicative of a close relationship at the genus level. A $\log$ (score) $\geq 2.0$ was set as the threshold for a match at the species level. Isolates with a $\log$ (score) $\geq 2.0$ were accepted as the correct identification.

Bacteria, media, and culture. Lactobacillus reuteri strains KCTC 3594, KCTC 3678, KCTC 3679, KCTC 3680, KCTC 3682, and KCTC 3683 were used as reference strains. All samples were purchased from the Korean Collection for Type Cultures (KCTC, Daejeon, Korea). Reference strains and newly isolated L. reuteri strains (AN417, AN306, AN403, AN413, AN507, AN509, AN510, AN511, AN513, AN519, AN523, AN705, AN711, and RI-7) were grown on MRS agar plates. P. gingivalis strain BAA-308 and F. nucleatum KCTC 15573 were purchased from the American Type Culture Collection (Manassas, VA, USA) and KCTC, respectively. They were grown in trypticase soy broth (TSB; BD, Germany) comprised of (per L): 30 g trypticase soy broth, $5 \mathrm{mg}$ hemin, $5 \mathrm{~g}$ yeast extract, $1 \mathrm{mg}$ vitamin $\mathrm{K} 1$, and $15 \mathrm{~g}$ agar, or blood TSB agar (TSB medium plus $15 \mathrm{~g} / \mathrm{L}$ agar and supplemented with $3 \%$ sheep blood). For all experiments, $P$. gingivalis and $F$. nucleatum were also cultured in TSB broth for at least $12 \mathrm{~h}$ prior to inoculation. Bacteria were grown and maintained at $37^{\circ} \mathrm{C}$ in an anaerobic chamber in an atmosphere of $\mathrm{CO}_{2}: \mathrm{H}_{2}: \mathrm{N}_{2}(5: 10: 85)$. S. mutans (KCTC 3065) strains were grown in brain heart infusion (BHI) medium at $37^{\circ} \mathrm{C}$ under aerobic conditions.

Preparation of the test sample. Lactobacillus reuteri AN417 was grown in MRS broth for $48 \mathrm{~h}$ at $37^{\circ} \mathrm{C}$ under anaerobic conditions to produce stationary phase cultures. The supernatant from L. reuteri AN417 culture (LRS) was collected via centrifugation at $4{ }^{\circ} \mathrm{C}$ for $30 \mathrm{~min}$ at $1500 \times \mathrm{g}$. The LRS was filtered through a $0.2 \mu \mathrm{m}$ membrane filter to remove the remaining bacteria and debris. L. reuteri AN417 cell extract (BE) was prepared by the addition of $0.3 \mathrm{~L}$ ethyl acetate to the cell pellet, stirring the mixture for $24 \mathrm{~h}$, and passing the mixture through a Phenex Teflon Polytetrafluorethylene filter membrane. After centrifugation at $1500 \times g$ for $20 \mathrm{~min}$, the cell pellet was removed. The cell-free supernatant and BEs were concentrated to $20 \times$ using a rotary evaporator and stored at $4{ }^{\circ} \mathrm{C}$ until required.

Determination of 1, 3-PDO production. Lactobacillus reuteri strains were grown in MRS medium (10 g/L proteose peptone No. 3, $10 \mathrm{~g} / \mathrm{L}$ beef extract, $5 \mathrm{~g} / \mathrm{L}$ yeast extract, $20 \mathrm{~g} / \mathrm{L}$ dextrose, $1 \mathrm{~g} / \mathrm{L}$ polysorbate 80 , $2 \mathrm{~g} / \mathrm{L}$ ammonium citrate, $5 \mathrm{~g} / \mathrm{L}$ sodium acetate, $0.2 \mathrm{~g} / \mathrm{L} \mathrm{MgSO}_{4}, 0.05 \mathrm{~g} / \mathrm{L} \mathrm{MnSO}_{4}$, and $2 \mathrm{~g} / \mathrm{L}$ dipotassium sulfate) supplemented with $20 \mathrm{~g} / \mathrm{L}$ glycerol for $12 \mathrm{~h}$ under anaerobic conditions. The concentration of 1,3-PDO was determined using a 1200 series HPLC system (Agilent, Santa Clara, CA, USA) with a refractive index detector (RID) and an ion-exchange column (Aminex HPX-87H; Bio-Rad, Hercules, CA, USA). The mobile phase was $2.5 \mathrm{mM} \mathrm{H}_{2} \mathrm{SO}_{4}$, flow rate was $0.6 \mathrm{~mL} / \mathrm{min}$, column temperature was $65^{\circ} \mathrm{C}$, and RID was maintained at $45^{\circ} \mathrm{C}$.

Measurement of the concentration of reuterin (3-HPA). Cultivation to stationary phase was performed in a $250 \mathrm{~mL}$ round-bottom flask containing $100 \mathrm{~mL}$ of MRS medium for $24 \mathrm{~h}$ at $37^{\circ} \mathrm{C}$. Cells were harvested by centrifugation at $16,000 \times g$ for $5 \mathrm{~min}$ and washed twice in $50 \mathrm{mM}$ sodium phosphate buffer (pH 7.5). The washed cells were resuspended in $200 \mathrm{mM}$ glycerol solution and incubated at $30^{\circ} \mathrm{C}$ for 3-HPA production. To measure the 3-HPA conversion yield, samples were collected at $1 \mathrm{~h}$ intervals. The samples were centrifuged at $16,000 \times g$ for $5 \mathrm{~min}$ and the supernatant was used to determine 3-HPA content. A previously described colorimetric method ${ }^{38}$ was used to determine the content of $3-\mathrm{HPA}$ in the samples. The reaction consisted of $1 \mathrm{~mL}$ of the sample, $3 \mathrm{~mL}$ of $\mathrm{HCl}(37 \%)$, and $0.75 \mathrm{~mL}$ of $10 \mathrm{mM}$ tryptophan-HCl. The mixture was mixed and incubated at $37^{\circ} \mathrm{C}$ for $20 \mathrm{~min}$, and the absorbance at $560 \mathrm{~nm}$ was measured using a SpectraMax 190 ELISA reader (Molecular Devices Corp., Sunnyvale, CA, USA). The amount of 3-HPA was calculated from the absorbance at $560 \mathrm{~nm}$ with acrolein as a standard. 
Disk diffusion assay. Antimicrobial activity was determined using the disk diffusion assay. Cell-free supernatants of $L$. reuteri strains were prepared using 48-h cultures. Agar plates were inoculated with E. coli, P. aeruginosa, or $S$. mutans. Filter paper discs, approximately $6 \mathrm{~mm}$ in diameter, soaked with $50 \mu \mathrm{L}$ of supernatant, were placed on the agar surface. After $24-48 \mathrm{~h}$ of incubation, the absence or presence of a clear zone around the disk was observed.

Growth rate measurement. To study the growth rates of the selected pathogenic bacteria in the presence or absence of LRS, bacteria were grown in $1 \mathrm{~mL}$ of the appropriate medium containing different concentrations of LRS at $37^{\circ} \mathrm{C}$ in an anaerobic chamber or orbital shaker. Growth rates were determined by measuring the culture optical density at $600 \mathrm{~nm}\left(\mathrm{OD}_{600}\right)$ at various times.

ATP bioluminescence assay. ATP levels in cultures of the selected pathogenic bacteria treated with various concentrations of LRS were measured using a BacTiter-Glo ATP Assay Kit (Promega, Madison, WI, USA) according to the manufacturer's instructions. Bioluminescence measurements were obtained in triplicate for each sample. Pure culture media were used as negative controls. Luminescence was measured using a luminometer (Promega).

Minimal inhibitory volume (MIV) assay. The MIV of LRS was determined using a modified method of a previously described procedure ${ }^{39}$. Pathogenic bacterial cultures generated in suitable liquid culture media as detailed above were diluted with nutrient broth to an $\mathrm{OD}_{600}$ of 0.005 or 0.05 . The diluted bacterial suspensions were then treated with either MRS medium (control) or $1 \times$ LRS, dispensed into the first well of a 96-well plate, and serially diluted into consecutive wells. Plates were incubated at $37^{\circ} \mathrm{C}$ for $24 \mathrm{~h}$, and the absorbance at $600 \mathrm{~nm}$ was measured using a microplate reader.

LIVE/DEAD BacLight viability assay. The LIVE/DEAD BacLight Kit (Molecular Probes-Invitrogen, Carlsbad, CA, USA) was used to distinguish live and dead bacterial cells. The assay, which is based on membrane integrity and nucleic acid staining, was performed according to the manufacturer's instructions. SYTO9 green fluorochrome (Thermo Fischer Scientific, Waltham, MA, USA) can penetrate the intact bacterial membrane, whereas the larger red fluorochrome, propidium iodide, only penetrates the membranes of damaged bacteria. Cells treated with LRS or MRS medium (control) for $24 \mathrm{~h}$ were stained in the dark for $15 \mathrm{~min}$. The cells were mounted on slides and evaluated by fluorescence microscopy.

Biofilm inhibition assay. The bacterial suspension was prepared by diluting an overnight culture of bacteria in TSB broth. Dilutions of LRS in bacterial suspensions were prepared in polystyrene-coated flat-bottomed 24-well plates and incubated at $37^{\circ} \mathrm{C}$ for 5 days without shaking to allow the development of multilayer biofilms. A pure culture medium served as a negative control. Biofilm biomass was assayed using the modified crystal violet staining assay and a LIVE/DEAD BacLight Kit (Molecular Probes-Invitrogen, Carlsbad, CA, USA) assay.

Time-kill assay. The time-to-kill $P$. gingivalis following LRS treatment was determined based on a previously described protocol ${ }^{40}$. A bacterial suspension $\left(\mathrm{OD}_{600}=1\right)$ was treated with LRS and incubated anaerobically at $37^{\circ} \mathrm{C}$. An aliquot $(100 \mu \mathrm{L})$ of this bacterial culture was removed at $0,4,8,24,48$, and $72 \mathrm{~h}$ following LRS treatment, and plated on TSB agar to quantify the number of colony forming units (CFUs) in the treated cultures. Pure MRS medium was used as a negative control.

Real-time quantitative polymerase chain reaction (RT-qPCR) analysis. The potential of LRS to prevent biofilm formation and/or destroy established biofilms was investigated. $P$. gingivalis $\left(\mathrm{OD}_{600}=0.6\right)$ was dispensed into a polystyrene-coated 6-well plate and treated with MRS medium (control) or LRS for $24 \mathrm{~h}$ in an anaerobic incubator. In addition, $P$. gingivalis biofilms were established in a polystyrene-coated 6-well plate for 5 days, after which these biofilms were treated with either MRS medium (control) or LRS for $48 \mathrm{~h}$ in an anaerobic incubator. Total RNA was extracted with TRIzol reagent, and cDNA was synthesized using a PrimeScript RT reagent Kit (TaKaRa Bio, Shiga, Japan). RT-qPCR analysis of cDNA was performed according to the manufacturer's instructions (Bioneer, Daejeon, South Korea) to investigate the mRNA expression of the selected genes related to biofilm formation. The following primers were used: hagA, Forward: 5'-ACAGCATCAGCCGATATT CC-3', Reverse: 5'-CGAATTCATTGCCACCTTCT-3'; hagB, Forward: 5'-TGTCGCACGGCAAATATCGCT AAAC-3', Reverse: 5'-CTGGCTGTCCTCGTCGAAAGCATAC-3'; rgpA, Forward: 5'-GCCGAGATTGTT CTT GAAGC-3', Reverse: 5'-AGGAGCAGCAATTGCAAAG-3'; $r g p$ B, Forward: 5'-CGCTGATGAAACGAACTT GA-3', Reverse: 5'-CTTCGAATACCATGCGGT-3'; kgp, Forward: 5'-AGCTGACAAAGGTGGAGACCAAAG G-3', Reverse: 5'-TGTGGCATG AGTTTTTCGGAACCGT-3'; and 16S rRNA, Forward: 5'-TGTAGATGACTG ATGGTG AAA-3', Reverse: 5'-ACTGTTAGCAACTACCGATGT-3'. The reaction procedure involved incubation at $95^{\circ} \mathrm{C}$ for $5 \mathrm{~min}$ followed by 30 cycles of $95^{\circ} \mathrm{C}$ for $10 \mathrm{~s}, 55^{\circ} \mathrm{C}$ for $30 \mathrm{~s}$, and $72^{\circ} \mathrm{C}$ for $30 \mathrm{~s}$. Three independent reactions were conducted in triplicate for each gene.

Effect of enzymes on antibacterial activity. Lactobacillus reuteri AN417 cell-free supernatants were treated with enzymes to evaluate the effect of enzymes on antibacterial substances. The LRS were treated with proteinase $\mathrm{K}(1 \mathrm{mg} / \mathrm{mL})$, lipase (700 units/mg), or $\alpha$-amylase (150 units/mg). For lipase and $\alpha$-amylase treatment, the $\mathrm{pH}$ of the LRS was adjusted to 6.5 with $\mathrm{NaOH}$ to facilitate enzymatic activity. Enzymes were activated by incubation of the enzyme-treated supernatant at $37^{\circ} \mathrm{C}$ for $3 \mathrm{~h}$, after which the enzymes were immediately 
inactivated at $95{ }^{\circ} \mathrm{C}$ for $3 \mathrm{~min}$. LRS-enzyme suspensions were centrifuged, and the supernatant was stored at $4{ }^{\circ} \mathrm{C}$ until further use.

Whole genome sequencing and analysis. Whole genome sequencing of strain 417 was performed using PacBio RS II (Pacific Biosciences, Menlo Park, CA, USA) SMRT sequencing technology. A standard PacBio library with an average of $20 \mathrm{~kb}$ inserts was prepared and sequenced, yielding $>371.74 \times$ average genome coverage. De novo assembly of the 128,664 subreads with 7,806 nucleotides on average $(1,004,367,686$ bp in total) was conducted using the hierarchical genome-assembly process pipeline in SMRT Analysis v2.3.0 ${ }^{41}$. To correct the sequencing errors that can occur at both ends of a contig, the SMRT resequencing protocol was performed with assembly in which the first half of the contig was switched with the second half. Protein-coding genes were predicted using Prodigal v.2.6.3. Ribosomal RNA, transfer RNA, and miscellaneous features were predicted using Rfam v12.0 ${ }^{42}$. CRISPR loci were predicted using the CRISPR recognition tool. ANI values were calculated using an online ANI calculator ${ }^{43}$.

Statistical analyses. All statistical analyses were performed using Student's t-test. The results are expressed as the mean \pm standard deviation for each group. Multiple group data were analyzed using one-way analysis of variance, followed by Dunnett's multiple range test. The threshold for significance was set at $p<0.05$. Data shown are representative of three independent experiments, except for Fig. 1B, in which the data are from two independent experiments.

\section{Data availability}

Whole genome sequences were deposited with Bioproject PRJNA637956 and Biosample SAMN15162791, respectively. GenBank accession numbers are CP054657 for single chromosome and CP054658-CP054661 for the four plasmids, respectively.

Received: 14 July 2020; Accepted: 28 December 2020

Published online: 15 January 2021

\section{References}

1. Hoare, A., Soto, C., Rojas-Celis, V. \& Bravo, D. Chronic inflammation as a link between periodontitis and carcinogenesis. Mediat. Inflamm. 2019, 1029857. https://doi.org/10.1155/2019/1029857 (2019).

2. Takenaka, S., Ohsumi, T. \& Noiri, Y. Evidence-based strategy for dental biofilms: Current evidence of mouthwashes on dental biofilm and gingivitis. Jpn. Dent. Sci. Rev. 55, 33-40 (2019).

3. Kononen, E., Gursoy, M. \& Gursoy, U. K. Periodontitis: A multifaceted disease of tooth-supporting tissues. J. Clin. Med. 8, 1135. https://doi.org/10.3390/jcm8081135 (2019).

4. Seguier, S., Gogly, B., Bodineau, A., Godeau, G. \& Brousse, N. Is collagen breakdown during periodontitis linked to inflammatory cells and expression of matrix metalloproteinases and tissue inhibitors of metalloproteinases in human gingival tissue?. J. Periodontol. 72, 1398-1406 (2001).

5. Tanner, A. C. Anaerobic culture to detect periodontal and caries pathogens. J. Oral. Biosci. 57, 18-26 (2015).

6. Shaddox, L. M. et al. Local inflammatory markers and systemic endotoxin in aggressive periodontitis. J. Dent. Res. 90, 1140-1144 (2011).

7. Mysak, J. et al. Porphyromonas gingivalis: Major periodontopathic pathogen overview. J. Immunol. Res. 2014, 476068. https://doi. org/10.1155/2014/476068 (2014).

8. Signat, B., Roques, C., Poulet, P. \& Duffaut, D. Fusobacterium nucleatum in periodontal health and disease. Curr. Issues Mol. Biol. 13, 25-36 (2011).

9. Jain, S. \& Darveau, R. P. Contribution of Porphyromonas gingivalis lipopolysaccharide to periodontitis. Periodontology 2000(54), 53-70 (2010).

10. Krzysciak, W., Jurczak, A., Koscielniak, D., Bystrowska, B. \& Skalniak, A. The virulence of Streptococcus mutans and the ability to form biofilms. Eur. J. Clin. Microbiol. Infect. Dis. 33, 499-515 (2014).

11. Li, X., Kolltveit, K. M., Tronstad, L. \& Olsen, I. Systemic diseases caused by oral infection. Clin. Microbiol. Rev. 13, 547-558 (2000).

12. Gorbach, S. L. Lactic acid bacteria and human health. Ann. Med. 22, 37-41 (1990).

13. Cardoso, R. S. et al. Effects of Bifidobacterium animalis subsp. lactis HN019 on ligature-induced periodontitis in rats with experimental rheumatoid arthritis. Benef. Microbes 11, 33-46 (2020).

14. Khalil, D., Hultin, M., Rashid, M. U. \& Lund, B. Oral microflora and selection of resistance after a single dose of amoxicillin. Clin. Microbiol. Infect. 22, 949e1-949e4. https://doi.org/10.1016/j.cmi.2016.08.008 (2016).

15. Lien, H. M. et al. Antimicrobial activity of Antrodia camphorata extracts against oral bacteria. PLoS ONE 9, e105286. https://doi. org/10.1371/journal.pone.0105286 (2014).

16. More, G., Tshikalange, T. E., Lall, N., Botha, F. \& Meyer, J. J. Antimicrobial activity of medicinal plants against oral microorganisms. J. Ethnopharmacol. 119, 473-477 (2008).

17. Choi, H. A. et al. Antimicrobial and anti-biofilm activities of the methanol extracts of medicinal plants against dental pathogens Streptococcus mutans and Candida albicans. J. Microbiol. Biotechnol. 27, 1242-1248 (2017).

18. Uraz, A., Karaduman, B., Isler, S. C., Gonen, S. \& Cetiner, D. Ozone application as adjunctive therapy in chronic periodontitis: Clinical, microbiological and biochemical aspects. J. Dent. Sci. 14, 27-37 (2019).

19. Haukioja, A. Probiotics and oral health. Eur. J. Dent. 4, 348-355 (2010).

20. Lim, H. S., Yeu, J. E., Hong, S. P. \& Kang, M. S. Characterization of antibacterial cell-free supernatant from oral care probiotic Weissella cibaria, CMU. Molecules 23, 1984 (2018).

21. Geraldo, B. M. C. et al. Heat-killed Lactobacillus reuteri and cell-free culture supernatant have similar effects to viable probiotics during interaction with Porphyromonas gingivalis. J. Periodontal. Res. 55, 215-220 (2020).

22. Wegh, C. A. M., Geerlings, S. Y., Knol, J., Roeselers, G. \& Belzer, C. Postbiotics and their potential applications in early life nutrition and beyond. Int. J. Mol. Sci. 20, 4673. https://doi.org/10.3390/ijms20194673 (2019).

23. Ren, D., Zhu, J., Gong, S., Liu, H. \& Yu, H. Antimicrobial characteristics of lactic acid bacteria isolated from homemade fermented foods. Biomed. Res. Int. 2018, 5416725. https://doi.org/10.1155/2018/5416725 (2018).

24. Craney, A., Ahmed, S. \& Nodwell, J. Towards a new science of secondary metabolism. J. Antibiot. (Tokyo) 66, 387-400 (2013) 
25. Bungenstock, L., Abdulmawjood, A. \& Reich, F. Evaluation of antibacterial properties of lactic acid bacteria from traditionally and industrially produced fermented sausages from Germany. PLoS ONE 15, e0230345. https://doi.org/10.1371/journal.pone.02303 45 (2020).

26. Wasfi, R., Abd El-Rahman, O. A., Zafer, M. M. \& Ashour, H. M. Probiotic Lactobacillus sp. inhibit growth, biofilm formation and gene expression of caries-inducing Streptococcus mutans. J. Cell Mol. Med. 22, 1972-1983 (2018).

27. How, K. Y., Song, K. P. \& Chan, K. G. Porphyromonas gingivalis: An overview of periodontopathic pathogen below the gum line. Front. Microbiol. 7, 53. https://doi.org/10.3389/fmicb.2016.00053 (2016).

28. Bjarnsholt, T. The role of bacterial biofilms in chronic infections. APMIS Suppl. 121, 1-51 (2013).

29. Saini, R., Saini, S. \& Sharma, S. Biofilm: A dental microbial infection. J. Nat. Sci. Biol. Med. 2, 71-75 (2011).

30. Jayathilake, P. G. et al. Extracellular polymeric substance production and aggregated bacteria colonization influence the competition of microbes in biofilms. Front. Microbiol. 8, 1865. https://doi.org/10.3389/fmicb.2017.01865 (2017)

31. Guilhen, C., Forestier, C. \& Balestrino, D. Biofilm dispersal: Multiple elaborate strategies for dissemination of bacteria with unique properties. Mol. Microbiol. 105, 188-210 (2017).

32. Kang, M. S. et al. Inhibitory effect of Lactobacillus reuteri on periodontopathic and cariogenic bacteria. J. Microbiol. 49, 193-199 (2011).

33. Cleusix, V., Lacroix, C., Vollenweider, S., Duboux, M. \& Le Blay, G. Inhibitory activity spectrum of reuterin produced by Lactobacillus reuteri against intestinal bacteria. BMC Microbiol. 7, 101. https://doi.org/10.1186/1471-2180-7-101 (2007).

34. Mu, Q., Tavella, V. J. \& Luo, X. M. Role of Lactobacillus reuteri in human health and diseases. Front. Microbiol. 9, 757. https://doi. org/10.3389/fmicb.2018.00757 (2018).

35. Khalaf, H. et al. Antibacterial effects of Lactobacillus and bacteriocin PLNC8 alphabeta on the periodontal pathogen Porphyromonas gingivalis. BMC Microbiol. 16, 188. https://doi.org/10.1186/s12866-016-0810-8 (2016).

36. Chun, J. \& Goodfellow, M. A phylogenetic analysis of the genus Nocardia with 16S rRNA gene sequences. Int. J. Syst. Bacteriol. 45, 240-245 (1995).

37. Yoon, S. H. et al. Introducing EzBioCloud: A taxonomically united database of 16S rRNA gene sequences and whole-genome assemblies. Int. J. Syst. Evol. Microbiol. 67, 1613-1617 (2017).

38. Slininger, P. J., Bothast, R. J. \& Smiley, K. L. Production of 3-hydroxypropionaldehyde from glycerol. Appl. Environ. Microbiol. 46, 62-67 (1983).

39. Spinler, J. K. et al. Human-derived probiotic Lactobacillus reuteri demonstrate antimicrobial activities targeting diverse enteric bacterial pathogens. Anaerobe 14, 166-171 (2008).

40. Appiah, T., Boakye, Y. D. \& Agyare, C. Antimicrobial activities and time-kill kinetics of extracts of selected Ghanaian mushrooms. Evid. Based Complement Alternat. Med. 2017, 4534350. https://doi.org/10.1155/2017/4534350 (2017).

41. Chin, C. S. et al. Nonhybrid, finished microbial genome assemblies from long-read SMRT sequencing data. Nat. Methods 10, 563-569 (2013).

42. Griffiths-Jones, S., Bateman, A., Marshall, M., Khanna, A. \& Eddy, S. R. Rfam: An RNA family database. Nucleic Acids Res. 31, 439-441 (2003).

43. Kook, J. K. et al. Genome-based reclassification of Fusobacterium nucleatum subspecies at the species level. Curr. Microbiol. 74, 1137-1147 (2017).

\section{Acknowledgements}

This work was supported by a National Research Foundation of Korea (NRF) Grant funded by the Korean Government (MSIP) (No. 2019R1F1A1051382 and No. 2016M3A9A5919255).

\section{Author contributions}

K.M.Y., J.-S.K., H.-W.J., D.-S.P. and K.-H..B conceived and designed the experiments. K.M.Y. and J.-S.K. analyzed the data. K.M.Y., D.-S.P. and K.-H.B. wrote the manuscript. H.-S.K., Y.-Y.K. and J.-K.O. provided help with critical discussion, editing, and final approval of the manuscript.

\section{Competing interests}

The authors declare no competing interests.

\section{Additional information}

Supplementary Information The online version contains supplementary material available at https://doi. org/10.1038/s41598-020-80921-X.

Correspondence and requests for materials should be addressed to D.-S.P. or K.-H.B.

Reprints and permissions information is available at www.nature.com/reprints.

Publisher's note Springer Nature remains neutral with regard to jurisdictional claims in published maps and institutional affiliations.

(c) (i) Open Access This article is licensed under a Creative Commons Attribution 4.0 International License, which permits use, sharing, adaptation, distribution and reproduction in any medium or format, as long as you give appropriate credit to the original author(s) and the source, provide a link to the Creative Commons licence, and indicate if changes were made. The images or other third party material in this article are included in the article's Creative Commons licence, unless indicated otherwise in a credit line to the material. If material is not included in the article's Creative Commons licence and your intended use is not permitted by statutory regulation or exceeds the permitted use, you will need to obtain permission directly from the copyright holder. To view a copy of this licence, visit http://creativecommons.org/licenses/by/4.0/.

(c) The Author(s) 2021 